Alma Mater Studiorum - Università di Bologna DEPARTMENT OF ECONOMICS

\title{
Competition and Screening with Skilled and Motivated Workers
}

Francesca Barigozzi

Nadia Burani

Quaderni - Working Paper DSE N 953

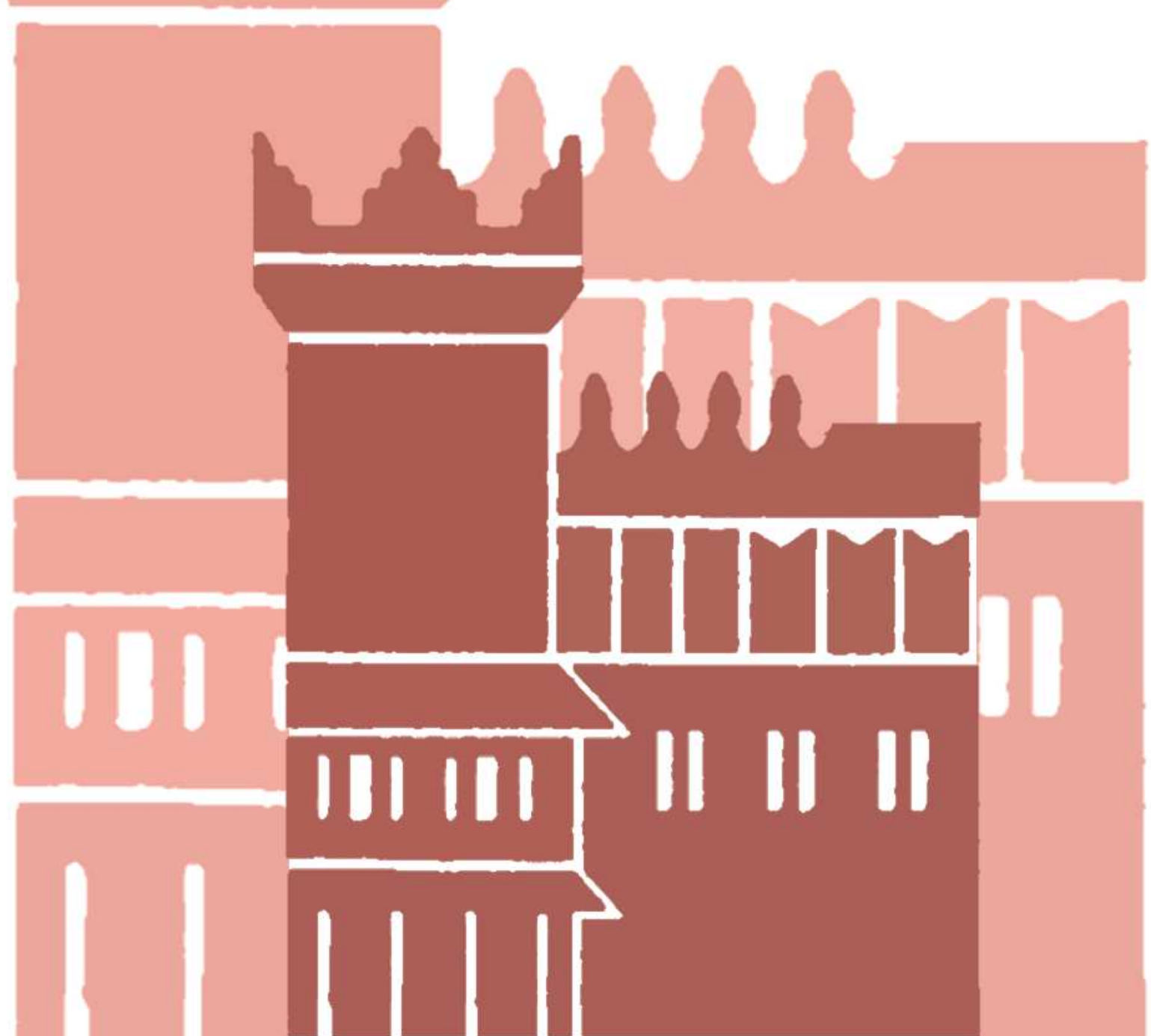




\title{
Competition and Screening with Skilled and Motivated Workers
}

\author{
Francesca Barigozzi* and Nadia Burani ${ }^{\dagger}$ \\ University of Bologna
}

June 2014

\begin{abstract}
We study optimal contracts offered by two firms competing for the exclusive services of one worker, who is privately informed about her ability and her motivation. Firms differ both in their production technology and in the mission they pursue and a motivated worker is keen to be hired by the missionoriented firm. We find that the matching of worker types to firms is always Pareto-efficient. When the difference in firms' technology is high, only the most efficient firm is active. When the difference is not very high, then agent types sort themselves by motivation: the mission-oriented firm hires motivated types and the profit-oriented firm employs non-motivated ones, independently of ability. Effort provision is higher when the worker is hired by the mission-oriented firm, but a compensating wage differential might exist: the motivated worker is paid less by the mission-oriented firm. Such an earnings penalty is driven entirely by motivation, is increasing in ability and is associated to low power of incentives.
\end{abstract}

Jel classification: D82, D86, J31, M55.

Key-words: vocational labor market, multi-principals, bidimensional screening, intrinsic motivation, skills.

\section{Introduction}

There exists a well-established empirical evidence, in the labor economics literature, on compensating wage differentials generated by differences in job characteristics or attributes for which heterogeneous workers have different willingnesses to pay (see Rosen, 1986). In turn, these idiosyncratic willingnesses to pay are reflected in wage differences across sectors . In particular, the existence of a compensating wage

\footnotetext{
${ }^{*}$ Department of Economics, University of Bologna, P.zza Scaravilli 2, 40126 Bologna (Italy). E-mail: francesca.barigozzi@unibo.it

${ }^{\dagger}$ Department of Economics, University of Bologna, Strada Maggiore 45, 40125 Bologna (Italy). E-mail: nadia.burani@unibo.it
} 
differential between the public and private sectors has been documented by Disney and Gosling (1998) and Melly (2005) among others. Similarly, Mocan and Tekin (2003), Preston (1989) and Gregg et al. (2011) show that average wages are lower in the not-for-profit sector relative to the for-profit one.

The idea that intrinsic motivation for being employed in the public or in the not-for-profit sectors (in general, in mission-oriented industries) might be the source of wage gaps has been proposed by a recent theoretical literature: see Handy and Katz (1998) for non-profit institutions vs corporations, Heyes (2005) for vocational vs non-vocational jobs and Delfgaauw and Dur (2007) that analyses applicants' tastes for being employed at a specific firm. A key prediction of this literature is that relatively low pay and weak monetary incentives endogenously emerge in sectors and jobs where intrinsic motivation matters. The role of heterogeneity in workers' productive ability is somehow neglected by the previous theoretical papers.

Nonetheless, another strand of empirical work points out that the compensating wage differential might arise because of a selection bias, given that a wage gap can also reflect unobservable differences in workers' ability across sectors (see Goddeeris 1988, Hwang, Reed and Hubbard 1992, Gibbons and Katz 1992, Goux and Maurin 1999).

Therefore, an open question still remains: are wages lower in mission-oriented sectors because of the lower reservation wage of motivated workers or because of the lower productivity of workers self-selecting into such sectors?

To this respect, Delfgaauw and Dur (2010) consider heterogeneity in both workers' productivity and motivation and show that the public-private earning differential comes partly from a compensating wage differential (motivated workers evaluate more being employed in the public sector) and partly is caused by selection arising endogenously from the adjustment in prices to differences in job attributes (on average more productive workers enter the private sector where remuneration is higher). This result holds when the demand for the public sector output is sufficiently low, and when motivation is unrelated to output. More importantly, they assume that firms perfectly observe both workers' characteristics.

However, when workers' productivity and motivation are the workers' private information, it becomes necessary to disentangle the pure wage differential from the selection effect of ability.

Previous results from theoretical literature admitting for workers' private information are ambiguous on whether vocation-based sectors are characterized by lower or higher workers' productivity on average. In particular, Handy and Katz (1998) assume that workers' productivity is identical across motivated and non-motivated workers. In Delfgaauw and Dur (2007), the impact of workers' productivity on the wage rate is ambiguous. Most importantly, Delfgaauw and Dur (2008) characterize the optimal incentive schemes offered by a public agency when workers differ in laziness (the opposite of productive ability) and public service motivation. They find that, when the public institution has to produce a low output then it only hires dedicated workers who are characterized by high ability and public service motivation. 
Conversely, when the public agency produces a sufficiently high output, ${ }^{1}$ then it attracts all dedicated as well as the laziest workers in the economy (i.e. the ones characterized by low ability and no vocation). However they underline that their "model does not necessarily imply that workers in the public sector are on average more lazy than workers in the private sector" (see page 173).

In our paper, we consider a labor market characterized by two sectors, a mission-oriented or vocational sector and a profit-oriented or standard sector. Each sector is represented by one firm only and the two firms compete to attract a single worker who is heterogeneous with respect to both her skills and her intrinsic motivation. These two characteristics are the worker's private information and are discretely, independently and uniformly distributed. The two firms offer screening contracts defined by a task level (the observable effort) and a non-linear wage rate. Because of competition and strategic interaction between the two firms, the worker's outside options are type-dependent and endogenous and thus we study a multi-principal framework with bidimensional screening.

Motivated workers care about the mission pursued by the firm for which they work. More precisely, the payoff of motivated agents depends on their own type but also on the type of firm hiring them. When a motivated worker is hired by the mission-oriented employer, and only by him, she benefits from intrinsic motivation and enjoys (at least to a certain extent) her personal contribution to the outcome produced by the firm. Conversely, all worker types experience a cost from effort provision when hired by an employer, which can differ across types but which does not depend on the type of employer. Thus, a peculiarity of our model is that the mission-oriented firm will have to design screening contracts based on both dimensions of private information, while the profit-oriented firm will have to offer the same contract to workers with the same ability level, taking into account that their outside options might differ depending on their intrinsic motivation.

The two firms' technologies are heterogeneous in that the marginal productivity of labor is different across sectors. When the difference in firms' technology is high, the most efficient firm succeeds in hiring all types of workers while the other firm is inactive.

When the difference in technology is not very high, then the mission-oriented firm has an advantage in hiring motivated types because of labor-donation aspect inherent in intrinsic motivation. This represents the most interesting case to analyze in fact, where agent types sort themselves by motivation: the mission-oriented firm hires motivated types and the profit-oriented firm employs non-motivated ones. Effort provision is almost always higher for motivated workers. Notably, with respect to our research question, our results show that workers' self-selection is ability-neutral. Indeed, we assume that the distribution of types is uniform, so that average ability is identical across sectors. This result would differ when alternative distributions of types are considered: if ability and intrinsic motivation are negatively correlated, then workers would again self-select according to motivation and average ability would be

\footnotetext{
${ }^{1}$ That is the opposite condition that is considered in Delfgaauw and Dur (2010).
} 
lower in the mission-oriented sector relative to the profit-oriented one.

We show that the allocation arising in equilibrium depends on the interplay between two different forces: the degree of competition on one side, determining the importance of workers' outside options, and the relevance of internal incentive compatibility on the other side. In particular, if competition is harsh, because firms are similar in technology and motivation is not too relevant, then both principals offer firstbest contracts to hired workers. In this case, outside options turn out to be more relevant than incentive compatibility and the equilibrium allocation is similar to the equilibrium with full information. Instead, if competition is mild, because firms are sufficiently different from each other, then both principals offer second-best contracts to hired workers. Here incentive compatibility is the driving force and equilibrium contracts are similar to the ones we observe under monopsony. Otherwise, when the degree of competition is neither harsh nor mild, we observe that one firm is relatively more efficient than the other and it optimally imposes lower distortions to less efficient types. To sum up, effort distortions are lower the higher the degree of competition between the two firms.

Moreover, competition reduces the set of worker's types that each firm can attract in equilibrium and this implies that the two firms need not resort to pooling contracts. Indeed, pooling is less pervasive for the mission-oriented firm than in the absence of competition. Our results on bidimensional screening extend the analysis of a companion paper, Barigozzi and Burani (2013), in which we fully characterized the optimal contracts offered by a monopsonistic mission-oriented firm.

For a wide range of parameter configurations, we find that a compensating wage differential emerges, in that, the total salary gained by a motivated type of worker in the vocational sector is lower than the salary that the same worker type would gain in the non-vocational sector. Such a wage penalty is particularly striking because it is always associated with higher effort provision on the part of motivated workers employed in the vocational sector. In addition, for a larger set of parameter values we also observe that equilibrium contracts offered by the mission-oriented firm are characterized by lower power of incentives than the ones designed by the standard firm; in different words, it is almost always the case that returns to skills are lower in the sector where motivation matters.

Interestingly, we are able to disentangle the effect of motivation and ability on the wage gap by observing that lower salaries in the vocational sector are driven by intrinsic motivation and not by ability. $^{2}$ However, ability does play a role in that the earnings penalty is increasing in ability. This result is consistent with the empirical evidence on the public-private wage gap documented in Roomking and Weisbrod (1999) and Bargain and Melly (2008), among others.

The rest of the paper is organized as follows. In the following subsection we describe the related

\footnotetext{
${ }^{2}$ Our discrete framework is particularly useful to study the wage differential because it allows us to compare the salary received by workers with the same ability in different sectors (with no need to recur to an average measure of ability in each sector).
} 
literature. In Section 2, we set up the model; in Section 3, the efficient solution is presented together with the equilibrium under full information. Section 4 describes the equilibrium screening strategies of the two principals when only one principal is able to hire all types of workers. In Section 5 , the characterization of the optimal contracts is provided when principals compete with each other and workers sort themselves by motivation. Subsection 5.4 comments on the existence of wage penalties in the mission-oriented sector and, finally, Section 6 concludes.

\section{$1.1 \quad$ Related literature}

Our work contributes to two different strands of literature: from an economic point of view, it adds to the recent and rapidly growing literature on the self-selection of workers with intrinsic motivation into different sectors of the labor market; from a technical point of view, it explicitly solves a multiprincipal game in a labor market where workers are characterized by two different dimensions of private information.

The problem of the design of optimal incentive schemes for intrinsically motivated workers has been tackled by Murdock (2002), Besley and Gathak (2005), whose attention has primarily been devoted to moral hazard, while we consider the screening problem.

Heyes (2005) and Delfgaauw and Dur (2007) are the first papers that address the issue of the selection of workers who are privately informed about their vocation. They show that, as a worker's motivation increases, the worker's reservation wage decreases. Therefore, as the wage increases, the average motivation of the workers who are willing to accept the job deteriorates.

Delfgaauw and Dur (2010) consider a richer framework where workers are heterogeneous with respect to both their intrinsic motivation and their ability. They focus on the issue of managerial self-selection into public vs private sectors under full information on the workers' characteristics: they argue that the return to managerial ability is always lower in the public than in the private sector, and that more able managers self-select into the private sector. They conclude that attracting a more able managerial workforce to the public sector by increasing remuneration up to the private sector levels is not efficient.

Our paper is also related to Handy and Katz (1998) and Delfgaauw and Dur (2008). The first paper shows that non-profits attract motivated managers by offering them compensation packages involving lower money wages and a larger component of institution-specific fringe benefits as compared to the private sector. But this result is driven by an exogenously given ranking of reservation wages for the different types of managers. Delfgaauw and Dur (2008) consider optimal contracts offered to civil servants who differ in laziness and public service motivation. They find that a cost-minimizing government optimally attracts dedicated as well as the laziest workers in the economy. We depart from this analysis in two main respects: first, their private sector is perfectly competitive and therefore firms do not interact 
strategically. Second, their screening mechanism is simplified because the public agency is constrained to hire at most two types of agents. ${ }^{3}$

More recently, Bénabou and Tirole (2013) analyze a model where firms compete to attract workers that are heterogeneous with respect to their productivity and their work ethics, i.e. the extent to which agents "do the right thing" beyond what their material self-interest commands. They show how competition for the most productive workers can interact with the incentive structure inside firms to undermine work ethics. The focus of Bénabou and Tirole (2013) is different from ours: they study the effects of firms' competition on the power of incentives schemes, we instead analyze the sorting of workers' types into mission-oriented and standard sectors. Moreover, they assume an affine compensation scheme with incentive power and a fixed wage, we instead consider non-linear contracts. Finally, in their framework with multitasking and moral hazard, screening is not bidimensional but it is performed by firms with respect to one dimension at a time (either productivity or work ethics).

From a technical point of view our paper draws both from the literature on multidimensional screening and from the literature on multi-principals. To the best of our knowledge, a model where both analyses are simultaneously carried out has not been studied before.

Screening when agents have several unobservable characteristics have been analyzed by some important papers that deal with continuous distribution of types: Armstrong and Rochet (1999), Armstrong (1996), Rochet and Chonè (1998), Basov (2001, 2005) and Deneckere and Severinov (2011). They all show that it is almost impossible to extend to the multidimensional environment the qualitative results and the regularity conditions of the unidimensional case. Our model is characterized by a discrete type space, and by one screening instrument available to the principal (namely the contractible effort level) so that the closest paper to ours is Armstrong (1999), which considers optimal price regulation of a monopoly that is privately informed about both its cost and demand function.

In the multi-principal literature (see for instance Stole 1995) the paper most closely related to us is Biglaiser and Mezzetti (1993) which studies two non-identical principals competing for the exclusive services of an agent in the presence of both adverse selection and moral hazard. The two principals have different technologies in that one principal is more efficient in hiring low skilled types while the other is more efficient in employing high skilled types. Intermediate types are the ones for whom competition is harsher: both principals make zero profits on these types, who get the same contract and are indifferent between working for either principal. Besides the difference between the continuous and the discrete setup, we depart from this work because we consider bidimensional rather than unidimensional screening.

\footnotetext{
${ }^{3}$ Barigozzi et al. (2013) and Barigozzi and Turati (2012) consider labor supply in a market where workers have private information on both productive ability and motivation. They show that the lemons' problem might be exacerbated by the presence of multidimensional asymmetric information because an increase in the market wage can determine a simultaneous decrease in both average vocation and average productivity of applicants.
} 


\section{The model}

We consider a multi-principal setting with bidimensional adverse selection. We assume that the economy is divided into two sectors, a non-vocational (or standard) sector, and a vocational (or mission-oriented) sector. In each sector operates a principal (he) willing to hire one agent (she) to perform a given task. The agent can work exclusively for one principal only. The principals and the agent are risk neutral.

Effort supplied by the agent is the only input the two firms need in order to produce. We call $e$ the observable and measurable effort (task) level that the agent is asked to provide. ${ }^{4}$ Both principals' production functions display constant returns to effort in such a way that

$$
q^{P}(e)=k^{P} e
$$

where $k^{P}$ denotes the marginal productivity of effort for principal $P \in\{V, N V\}$, with $V$ referring to the principal operating in the vocational-sector and $N V$ referring to the non-vocational principal. We normalize the marginal productivity of effort for the mission-oriented principal to $k^{V}=1$ and set $k^{N V}=$ $k .^{5}$

The principals' profit functions are given by

$$
\pi^{P}(e)=q^{P}(e)-w^{P}=k^{P} e-w^{P}
$$

where the price of output is assumed to be exogenous and normalized to 1 in both sectors, and where $w^{P}$ is the salary paid to the worker hired by principal $P$. Since the principals' profit only indirectly depends on the type of the agent, we are considering a setting with private values.

Suppose that the agent differs in two characteristics, productive ability and intrinsic motivation, that are independently and uniformly distributed.

We interpret worker's characterized by high ability as an agent incurring in a low cost of providing a given effort level. Workers can have only two possible levels of cost of effort provision $\theta_{i} \in\left\{\theta_{L}, \theta_{H}\right\}$, where $\theta_{H}>\theta_{L}>0$. High ability corresponds to a low cost of effort provision $\theta_{L}$, low ability to a high cost of effort provision $\theta_{H} \cdot{ }^{6}$

As for intrinsic motivation, we mainly refer to Delfgaauw and Dur (2008) and assume that workers, to a certain extent, derive utility from exerting effort in the mission-oriented sector. Since there exists a one-to-one relationship between effort exerted and output produced by the vocational principal, this

\footnotetext{
${ }^{4}$ In particular, the variable $e$ can be interpreted as a job-specific requirement like the amount of hours of labor the agent is asked to devote to production or the speed at which a production line is run in a factory.

${ }^{5}$ For the time being, we do not impose any constraints on the magnitude of $k^{N V}$, which can be smaller or greater than $k^{V}$.

${ }^{6}$ Note that, as usual, modelling one worker who can take four possible types with equal probability is equivalent to considering a unit mass set of workers divided into four different groups of types with the same frequency.
} 
interpretation is equivalent to considering intrinsic motivation as the enjoyment of one's personal contribution to the vocational principal's output. ${ }^{7}$ What matters is that the benefit from intrinsic motivation can only be enjoyed when a motivated worker is employed in the vocational sector. In different words, ability is the only relevant worker's characteristic in the non-vocational sector. Paralleling ability, we assume that motivation can take only two possible values $\gamma_{j} \in\left\{\gamma_{L}, \gamma_{H}\right\}$. A worker can have either high motivation $\gamma_{H}$, or low motivation $\gamma_{L}$.

So there are four types of agents denoted as $i j=\{L H, L L, H H, H L\}$ where the first index represents the cost of effort provision and the second motivation.

Without loss of generality, we normalize the lower bounds of the support of the distribution for both attributes, setting $\theta_{L}=1$ and $\gamma_{L}=0$. We will thus focus on situations in which the agent can be either intrinsically motivated, with motivation parameter taking value $\gamma_{H}=\gamma$ or not motivated at all. Our results will depend on how the difference or heterogeneity in motivation $\Delta \gamma=\gamma_{H}-\gamma_{L}=\gamma$ relates to the difference in ability $\Delta \theta=\theta_{H}-\theta_{L}=\theta-1$. Given our normalization, we will refer to the difference in motivation $\Delta \gamma$ and to the level of motivation $\gamma$ interchangeably. Furthermore, we impose that $\Delta \gamma \leq 1$ or else that $0<\gamma \leq 1$ and that $\Delta \theta \leq 1$ or else that $1<\theta \leq 2$ (these assumptions are made in order to avoid volunteer work and to allow low-skilled, motivated workers to be more productive than high-skilled, non-motivated agents at the first best, respectively).

The agent's reservation utility is endogenous and depends on the contract offered by the opponent principal. We assume that, if the worker is not hired by any principal, her utility is zero.

When a worker is hired by the non-vocational principal, her utility is

$$
U_{i j}^{N V}=w_{i j}-\frac{1}{2} \theta_{i} e_{i j}^{2}
$$

In fact, motivated workers do not enjoy any benefit from motivation when hired by the standard firm. As a consequence, from the point of view of the non-vocational principal, workers $L H$ and $L L$ on one side and workers $H H$ and $H L$ on the other side are equally productive. ${ }^{8}$

The single crossing condition is obviously always verified with respect to the ability parameter in the non-vocational sector because $M R S_{e, w}^{N V}=-\frac{\partial U_{i j}^{N V} / \partial e_{i j}}{\partial U_{i j}^{N V} / \partial w_{i j}}=\theta_{i} e_{i j}$ and $\frac{\partial M R S_{e, w}^{N V}}{\partial \theta_{i}}>0$.

When a worker is hired by the vocational principal, her utility takes the form

$$
U_{i j}^{V}=w_{i j}-\frac{1}{2} \theta_{i} e_{i j}^{2}+\gamma_{j} e_{i j}
$$

\footnotetext{
${ }^{7}$ The same interpretation of intrinsic motivation can be found in Besley and Ghatak (2005) and Delfgaauw and Dur (2007, 2008, 2010-only as for Section 5) and traces back to the "warm-glow giving" or impure altruism theory in Andreoni (1990).

${ }^{8}$ However, types of agent with the same ability potentially benefit from different outside options. In fact, given ability, motivated workers are more productive than non-motivated ones when employed by the vocational principal. In different words, intrinsic motivation affects the reservation utility but not the productivity of workers when they are employed in the non-vocational sector.
} 
where both productivity $\theta_{i}$ and motivation $\gamma_{j}$ are related to effort exertion. ${ }^{9}$

The marginal rate of substitution between effort and wage is given by

$$
\operatorname{MRS}_{e, w}^{V}=-\frac{\partial U_{i j}^{V} / \partial e_{i j}}{\partial U_{i j}^{V} / \partial w_{i j}}=\theta_{i} e_{i j}-\gamma_{j}
$$

which is always positive for non-motivated workers with $\gamma_{j}=0$. When the effort required by the principal is sufficiently high, i.e. when $e_{i j}>\frac{\gamma_{j}}{\theta_{i}}$, also motivated workers' indifference curves have the standard positive slope in the space $(e, w)$ and effort is a "bad".

Note that providing effort represents a net cost to the agent when

$$
-\frac{1}{2} \theta_{i} e_{i j}^{2}+\gamma_{j} e_{i j}<0
$$

Thus, if the effort required by the mission-oriented principal is sufficiently low, motivated workers could perform their task when receiving a non-positive reward, in other words they would be ready to volunteer to be hired in the mission-oriented sector.

Finally, notice that agents' utility function satisfies the single-crossing property, but only with respect to each parameter of private information at a time. ${ }^{10}$ This means that the indifference curves of types with the same motivation but different ability intersect only once at $e=0$, and the same is true for the indifference curves of workers endowed with the same ability but different motivation. Nonetheless, the indifference curves of intermediate types $H H$ and $L L$ cross twice, the second intersection occurring at $e=\frac{2 \gamma}{\theta-1}$.

Remark 1 In the mission-oriented sector, the single-crossing property does not hold.

In the mission-oriented sector, the combined impact of both ability and motivation on the worker's effort and on the firm's output is as follows: the most productive type is worker $L H$ (with low effort cost and high motivation) who is expected to exert the highest effort, whereas the least productive type is worker $H L$ (with high effort cost and no motivation) who is expected to provide the lowest effort. Worker types $L L$ and $H H$ are in-between and their effort levels cannot be ordered unambiguously. ${ }^{11}$

\footnotetext{
${ }^{9}$ This linear-quadratic specification of the utility function is widely used in the literature on workers' intrinsic motivation (see Besley and Ghatak 2005 and Delfgaauw and Dur 2010). The same objective function for the agent is also considered in the literature on multidimensional screening with a continuum of types (see Laffont et al. 1987, Basov 2005, and Deneckere and Severinov 2011), where solutions are found imposing that the type space be the unit square.

${ }^{10}$ All the properties of the utility function extend to the more general case in which the cost of effort is still convex while the benefit from exerting effort, due to intrinsic motivation, is concave.

${ }^{11}$ Notice that the existence of two possible orderings of effort levels is a consequence of the bidimensionality of our problem and of the failure of the single-crossing condition. It could not be generated in a unidimensional set-up with different types of employees characterized by a single summary statistic, like the overall cost of providing effort.
} 
The timing of the game is as follows. The two principals simultaneously offer the worker a menu of contracts of the form $\left\{e_{i j}^{P}, w^{P}\left(e_{i j}\right)\right\}$, with $P \in\{V, N V\}$. The worker observes the contracts, chooses which principal (if any) to work for and selects a contract. Then the worker exerts the effort level specified by the chosen contract, output is produced and the contracted wage is paid.

An equilibrium is such that each principal chooses a menu of contracts that maximizes his expected profit, given the contracts offered by the rival principal and given the equilibrium choice of the worker. The worker chooses the contract that maximizes her utility. The principals are bound to offer contracts that make non-negative profit. If a worker is indifferent between working for the two principals, it is assumed that with probability one such worker will choose to work for the principal making the higher profit. In fact the principal with the higher payoff is able to raise her reward by $\varepsilon>0$ and break the tie.

In Section 5 we will study competition with (bidimensional) adverse selection. Before turning to the second-best with competition, let us examine some benchmark cases.

\section{Benchmark cases}

In this section we illustrate the first-best of the model and the equilibrium under full information.

\subsection{The social planner}

Suppose that a social planner, endowed with perfect information about the applicant's characteristics, maximizes total surplus by assigning types of worker to principals. The first-best effort levels are obtained by maximizing total surplus (sum of the agent's utility and the principal's profit) with respect to the workers' effort for each type of worker and for each type of principal and are as follows

$$
e_{L H}^{F B, V}=1+\gamma \quad e_{H H}^{F B, V}=\frac{1+\gamma}{\theta} \quad e_{L L}^{F B, V}=1 \quad e_{H L}^{F B, V}=\frac{1}{\theta}
$$

for the vocational principal and

$$
e_{L H}^{F B, N V}=e_{L L}^{F B, N V}=e_{L}^{F B, N V}=k \quad e_{H H}^{F B, N V}=e_{H L}^{F B, N V}=e_{H}^{F B, N V}=\frac{k}{\theta}
$$

for the non-vocational principal.

When $1<k<1+\gamma$, the social planner assigns motivated workers to the vocational principal and non-motivated types of worker to the non-vocational principal. The labor market is fully segmented according to motivation, and workers' ability is evenly distributed across sectors.

When $k=1$, the two principals are equally productive in hiring non-motivated workers $L L$ and $H L$ but principal $V$ is more productive when employing motivated types $H H$ and $L H$, so that the vocational principal alone can guarantee efficiency. 
When $k=1+\gamma$, instead, the two principals are equally productive in hiring motivated workers $L H$ and $H H$ and principal $N V$ is more productive than the vocational principal when employing nonmotivated types $L L$ and $H L$, so that the non-vocational principal alone might efficiently hire all worker's types. Note that, for $k<1$, efficiency would require that the vocational principal is the unique employer, whereas for $k>1+\gamma$ efficiency would require that the non-vocational principal is the unique employer.

\subsection{Competition under full information}

Here the two principals observe the worker's type and simultaneously offer her a contract $\left(e_{i j}^{P}, w_{i j}^{P}\right)$, with $P \in\{V, N V\}$. The best strategy is to ask each agent the first best effort level: this allows the two principals to generate the highest revenue to be used to attract the worker (notice that the game describes a situation where two firms characterized by different efficiency levels compete à la Bertrand to attract a worker of known type).

The association of types to principals is the efficient one described in the previous subsection. When $1<k<1+\gamma$, the standard firm will make its highest offer to motivated workers, that is the first best total surplus (see expression 3 below for the wage that a worker receives when she is rewarded the first best total surplus) and the mission-oriented firm will meet that offer attracting motivated workers. ${ }^{12}$ In the same way and in the case of non-motivated workers, the mission-oriented firm will offer the first best total surplus and, the standard firm will meet that offer attracting the two worker's types.

When $k \leq 1$ and $k \geq 1+\gamma$ the more productive principal is hiring all the workers.

In Appendix B we compute the wages offered by the two principals in equilibrium, we further discuss the cases with $k=1$ and $k=1+\gamma$ and, summarize the properties of the allocation.

\section{Fully dominant principals}

We start tackling the issue of competition between the two non-informed principals in the extreme cases in which, in equilibrium, all worker's types are hired by one principal and the other principal remains inactive. Following Biglaiser and Mezzetti (1993), we call such situations of "deterred competition" equilibria with a fully dominant principal.

When a principal is fully dominant, he is able to hire all types of workers and to make non-negative profits on all types, even when the rival principal, who is called the dominated principal, offers them their first best total surplus. To be more precise, suppose that principal $P \in\{V, N V\}$ is the dominated principal. Then he unsuccessfully competes with the dominant principal by offering each type of worker a contract such that: $(i)$ the effort level is the first-best effort $e_{i j}^{F B, P}$, and (ii) the total wage is obtained

\footnotetext{
${ }^{12}$ Notice that any lower wage possibly offered by the less efficient principal would generate profitable deviations and thus cannot be an equilibrium strategy.
} 
imposing zero profits on that type, i.e.

$$
\pi_{i j}^{P}=k^{P} e_{i j}^{F B, P}-w_{i j}^{P}=0 \Longleftrightarrow w_{i j}^{P}=k^{P} e_{i j}^{F B, P} .
$$

In this way, the dominated principal offers each type of agent the maximal possible utility that he can, which is given by

$$
U_{i j}^{T S, P}=k^{P} e_{i j}^{F B, P}-\frac{1}{2} \theta_{i} e_{i j}^{F B, P}+\gamma_{j} e_{i j}^{F B, P},
$$

where the superscript $T S$ stands for total surplus utility and where the term $\gamma_{j} e_{i j}^{F B, P}$ is equal to zero when the dominated principal is the non-vocational one. Conversely, the fully dominant principal succeeds in attracting all types of worker by offering them at least $U_{i j}^{T S, P}$.

Intuitively, a principal is fully dominant when he is much more "productive" than the other, where by "productive" we mean the combination of marginal productivity of effort and intrinsic motivation and their joint effect on effort and output provision.

\subsection{Fully dominant non-vocational principal}

Recall that the non-vocational principal is only able to screen applicants on the basis of their ability and that intrinsic motivation does not play any role for the design of the optimal incentive scheme. In other words, any incentive compatible contract that the non-vocational principal might offer must be such that workers with the same ability are offered the same contract, whereby

$$
e_{L H}^{N V}=e_{L L}^{N V}=e_{L}^{N V} \text { and } e_{H H}^{N V}=e_{H L}^{N V}=e_{H}^{N V}
$$

and

$$
w_{L H}^{N V}=w_{L L}^{N V}=w_{L}^{N V} \text { and } w_{H H}^{N V}=w_{H L}^{N V}=w_{H}^{N V}
$$

But types characterized by the same ability and different intrinsic motivation are not completely identical to the non-vocational principal, because they enjoy different outside options or reservation utilities. Indeed, when the non-vocational principal is fully dominant, the workers' outside options are equal to their first best total surplus in the mission-oriented sector and given by

$$
U_{L H}^{T S, V}=\frac{(1+\gamma)^{2}}{2} \quad U_{H H}^{T S, V}=\frac{(1+\gamma)^{2}}{2 \theta} \quad U_{L L}^{T S, V}=\frac{1}{2} \quad U_{H L}^{T S, V}=\frac{1}{2 \theta}
$$

Note that $U_{L H}^{T S, V}>U_{L L}^{T S, V}$ and that $U_{H H}^{T S, V}>U_{H L}^{T S, V}$, namely that the vocational principal is always able to leave to motivated types a strictly higher utility than to non-motivated types with the same ability. Then, the fully dominant non-vocational principal's program is

$$
\max _{\left(e_{i \cdot}^{N V}, w_{i}^{N V}\right)} E\left(\pi^{N V}\right)=\frac{1}{2}\left[\left(k e_{L \cdot}^{N V}-w_{L}^{N V}\right)+\left(k e_{H}^{N V}-w_{H}^{N V}\right)\right]
$$


subject to the two participation constraints of motivated types ${ }^{13}$

$$
w_{i}^{N V}-\frac{1}{2} \theta_{i}\left(e_{i \cdot}^{N V}\right)^{2} \geq U_{i H}^{T S, V}
$$

for every $i=L, H$ and two incentive compatibility constraints

$$
w_{i \cdot}^{N V}-\frac{1}{2} \theta_{i}\left(e_{i \cdot}^{N V}\right)^{2} \geq w_{i^{\prime} .}^{N V}-\frac{1}{2} \theta_{i}\left(e_{i^{\prime} \cdot}^{N V}\right)^{2}
$$

for every $i=L, H$ and $i^{\prime} \neq i$. Adding the two incentive compatibility constraints, one can easily check that implementability requires that

$$
e_{L H}^{N V}=e_{L L}^{N V}=e_{L}^{N V}>e_{H H}^{N V}=e_{H L}^{N V}=e_{H}^{N V}
$$

In order to solve problem PNVFD, we build on the analysis of Martimort and Laffont (2002, Chapter 3.3, pages 101-105) which is devoted to the study of type-dependent participation constraints and countervailing incentives when there are only two types of agent and the inefficient type's outside option is zero while the efficient type's reservation utility is positive. As in Martimort and Laffont (2002), the solution exhibits five different regimes according to which participation and incentive compatibility constraints are binding. In turn, different constraints might be binding in our setup depending on how the difference in reservation utilities $U_{L H}^{T S, V}-U_{H H}^{T S, V}$ relates to the optimal effort levels associated to each regime (which are a function of the marginal productivity of effort in the non-vocational sector $k$ ).

Case 1 (Irrelevance of outside options) This case occurs when $P C_{H H}^{N V}$ and $I C_{L \cdot v s H}^{N V}$. are binding. In terms of effort levels, the solution is such that $e_{H}^{S B, N V}=\frac{k}{(2 \theta-1)}$ and $e_{L}^{S B, N V}=k=e_{L}^{F B, N V}$. Thus the second-best solution remains valid despite the type-dependent outside options. This case is relevant when $U_{L H}^{T S, V}-U_{H H}^{T S, V}<\frac{(\theta-1)}{2}\left(e_{H}^{S B, N V}\right)^{2}=\frac{(\theta-1) k^{2}}{2(2 \theta-1)^{2}}$.

Case 2 This case occurs when both $P C_{H}^{N V}$ and $P C_{L H}^{N V}$ and also $I C_{L \cdot v s H}^{N V}$. are binding. The solution is such that $e_{H}^{*, N V}=\sqrt{\frac{2\left(U_{L H}^{T S, V}-U_{H H}^{T S V}\right)}{\theta-1}}$ (which is in-between the second- and the first-best level) and $e_{L}^{F B, N V}=k$. This case is relevant when $\frac{(\theta-1)}{2}\left(e_{H}^{S B, N V}\right)^{2} \leq U_{L H}^{T S, V}-U_{H H}^{T S, V}<\frac{(\theta-1)}{2}\left(e_{H}^{F B, N V}\right)^{2}=$ $\frac{(\theta-1) k^{2}}{2 \theta^{2}}$.

Case 3 This case occurs when only $P C_{H H}^{N V}$ and $P C_{L H}^{N V}$ are binding. The solution is the first-best one, that is $e_{H}^{F B, N V}=\frac{k}{\theta}$ and $e_{L}^{F B, N V}=k$. This case is relevant when $\frac{(\theta-1)}{2}\left(e_{H}^{F B, N V}\right)^{2} \leq U_{L H}^{T S, V}-$ $U_{H H}^{T S, V}<\frac{(\theta-1)}{2}\left(e_{L}^{F B} \cdot, N V\right)^{2}=\frac{(\theta-1) k^{2}}{2}$.

Case 4 This case occurs when both $P C_{H H}^{N V}$ and $P C_{L H}^{N V}$ and $I C_{H \cdot v s L}^{N V}$. are binding. The solution is such that $e_{H}^{F B, N V}=\frac{k}{\theta}$ and $e_{L}^{*, N V}=\sqrt{\frac{2\left(U_{L H}^{T S, V}-U_{H H}^{T S, V}\right)}{\theta-1}}$ (which is in-between the first-best level and

\footnotetext{
${ }^{13}$ Given the magnitudes of $U_{i j}^{T S, V}$, only the participation constraints of motivated types matter. Indeed, once $P C_{L H}^{N V}$ is satisfied, then $P C_{L L}^{N V}$ is slack and, similarly, once $P C_{H H}^{N V}$ holds, then also $P C_{H L}^{N V}$ is satisfied with strict inequality.
} 
the countervailing incentives solution $e_{L}^{C I, N V}$ defined in the last case). This case is relevant when $\frac{(\theta-1)}{2}\left(e_{L}^{F B, N V}\right)^{2} \leq U_{L H}^{T S, V}-U_{H H}^{T S, V} \leq \frac{(\theta-1)}{2}\left(e_{L}^{C I, N V}\right)^{2}=\frac{(\theta-1) k^{2}}{2(2-\theta)^{2}}$.

Case 5 (Countervailing Incentives) This case occurs when $P C_{L H}^{N V}$ and $I C_{H \cdot v s L}^{N V}$. are binding. The solution is such that $e_{H}^{F B, N V}=\frac{k}{\theta}$ and $e_{L}^{C I, N V}=\frac{k}{(2-\theta)}$ (which is above both the first-best level and $\left.e_{L}^{*, N V}\right)$. This case is relevant when $U_{L H}^{T S, V}-U_{H H}^{T S, V} \geq \frac{(\theta-1)}{2}\left(e_{L}^{C I, N V}\right)^{2}$.

Since the difference in reservation utilities is fixed and equal to $U_{L H}^{T S, V}-U_{H H}^{T S, V}=\frac{(\theta-1)(1+\gamma)^{2}}{2 \theta}$, straightforward computations show that the only possible situations in which the non-vocational principal is fully dominant are Cases from 1 to 3 .

In particular, Case 1 holds for $k>\frac{(1+\gamma)(2 \theta-1)}{\sqrt{\theta}}=k_{1}$, Case 2 holds for $k_{2}=(1+\gamma) \sqrt{\theta}<k \leq k_{1}$ and Case 3 holds for $k_{3}=\frac{1+\gamma}{\sqrt{\theta}}<k \leq k_{2}$, where $k_{1}>k_{2}>1+\gamma>k_{3}$. But when $k<1+\gamma$ the nonvocational principal makes strictly negative profits. Hence, Case 3 is only relevant for $1+\gamma \leq k \leq k_{2}$, while the remaining regimes 4 and 5, which are associated with even lower levels of $k$ and with strictly negative profits, must be discarded. This fact implies that the non-vocational principal never resorts to countervailing incentives.

Also notice that, when $k=1+\gamma$, the non-vocational principal is in Case 3 and is able to hire all types of workers offering precisely the same contracts as the rival principal. Thus the non-vocational principal makes zero profits on all types, and the latter are indifferent between the two sectors. Such a situation cannot be an equilibrium because the non-vocational principal can profitably deviate by attracting all types except type $H H$, who strictly prefers the contract offered by the mission-oriented firm. When $k=1+\gamma$, Section 5.3 shows that the equilibrium is such that workers sort themselves by motivation and the non-vocational principal only hires non-motivated types making strictly positive profits on each potential applicant.

The above discussion can be summarized in the Proposition that follows.

Proposition 1 The non-vocational principal is fully dominant if $k>1+\gamma$.

\subsection{Fully dominant vocational principal}

When principal $V$ fully dominates, the equilibrium strategy of principal $N V$ is to make the agent the residual claimant so that she receives all the surplus. Therefore each type is asked to provide the first-best level of effort and the non-vocational principal makes zero profits on each type, who is left with her total surplus utility

$$
U_{L}^{T S, N V}=\frac{k^{2}}{2} \text { or } U_{H}^{T S, N V}=\frac{k^{2}}{2 \theta} .
$$

Note that such utilities only differ according to ability. 
Now, the vocational principal must offer each type of agent a level of utility at least as high as $U_{i}^{T S, N V}$ for $i=L, H$. Thus the vocational principal solves the maximization problem

$$
\max _{\left(e_{i j}^{V}, w_{i j}^{V}\right)} E\left(\pi^{V}\right)=\frac{1}{4}\left[\left(e_{L H}^{V}-w_{L H}^{V}\right)+\left(e_{L L}^{V}-w_{L L}^{V}\right)+\left(e_{H H}^{V}-w_{H H}^{V}\right)+\left(e_{H L}^{V}-w_{H L}^{V}\right)\right]
$$

subject to four participation constraints whose generic form is

$$
w_{i j}-\frac{1}{2} \theta_{i}\left(e_{i j}^{V}\right)^{2}+\gamma_{j} e_{i j}^{V} \geq U_{i}^{T S, N V}
$$

and twelve incentive compatibility constraints that are such that

$$
w_{i j}^{V}-\frac{1}{2} \theta_{i}\left(e_{i j}^{V}\right)^{2}+\gamma_{j} e_{i j}^{V} \geq w_{i^{\prime} j^{\prime}}^{V}-\frac{1}{2} \theta_{i}\left(e_{i^{\prime} j^{\prime}}^{V}\right)^{2}+\gamma_{j} e_{i^{\prime} j^{\prime}}^{V} \quad \quad\left(I C_{i j v s i^{\prime} j^{\prime}}^{V}\right)
$$

with $i j$ different from $i^{\prime} j^{\prime}$.

The solution to this program is found extending the analysis of a companion paper, Barigozzi and Burani (2013), where the problem of bidimensional screening is considered for type-independent reservation utilities (normalized to zero) and for a non-uniform distribution of the worker's types.

Note that $P C_{H H}^{V}$ is slack once $I C_{H H v s H L}^{V}$ and $P C_{H L}^{V}$ are both satisfied: indeed, the following chain of inequalities holds

$$
\underbrace{w_{H H}^{V}-\frac{1}{2} \theta\left(e_{H H}^{V}\right)^{2}+\gamma e_{H H}^{V} \geq w_{H L}^{V}-\frac{1}{2} \theta\left(e_{H L}^{V}\right)^{2}+\gamma e_{H L}^{V}}_{I C_{H H v s H}^{V}}>\underbrace{w_{H L}^{V}-\frac{1}{2} \theta\left(e_{H L}^{V}\right)^{2} \geq \frac{k^{2}}{2 \theta}}_{P C_{H L}^{V}}
$$

implying that

$$
w_{H H}^{V}-\frac{1}{2} \theta\left(e_{H H}^{V}\right)^{2}+\gamma e_{H H}^{V}>\frac{k^{2}}{2 \theta},
$$

which is precisely $P C_{H H}^{V}$ holding with strict inequality. Similarly, $P C_{L H}^{V}$ is slack once $I C_{L H v s L L}^{V}$ and $P C_{L L}^{V}$ both hold because

$$
\underbrace{w_{L H}^{V}-\frac{1}{2}\left(e_{L H}^{V}\right)^{2}+\gamma e_{L H}^{V} \geq w_{L L}^{V}-\frac{1}{2}\left(e_{L L}^{V}\right)^{2}+\gamma e_{L L}^{V}}_{I C_{L H v s L L}^{V}}>\underbrace{w_{L L}^{V}-\frac{1}{2}\left(e_{H L}^{V}\right)^{2} \geq \frac{k^{2}}{2}}_{P C_{L L}^{V}} .
$$

So one can consider a reduced program where the participation constraints of motivated types $P C_{L H}^{V}$ and $P C_{H H}^{V}$ are omitted.

Finally, concerning $P C_{L L}^{V}$ and $P C_{H L}^{V}$, one can write

$$
\underbrace{w_{L L}^{V}-\frac{1}{2}\left(e_{L L}^{V}\right)^{2} \geq w_{H L}^{V}-\frac{1}{2}\left(e_{H L}^{V}\right)^{2}}_{I C_{L L v s H L}^{V}}>\underbrace{w_{H L}^{V}-\frac{1}{2} \theta\left(e_{H L}^{V}\right)^{2} \geq \frac{k^{2}}{2 \theta}}_{P C_{H L}^{V}} .
$$

In order for $P C_{L L}^{V}$ to be satisfied when $P C_{H L}^{V}$ is, assume first that $P C_{H L}^{V}$ is binding and then substitute the corresponding expression for $w_{H L}^{V}$ into the right hand side of $I C_{L L v s H L}^{V}$. Then $P C_{L L}^{V}$ will be slack whenever

$$
w_{L L}^{V}-\frac{1}{2}\left(e_{L L}^{V}\right)^{2} \geq \frac{1}{2}(\theta-1)\left(e_{H L}^{V}\right)^{2}+\frac{k^{2}}{2 \theta}>\frac{k^{2}}{2}
$$


a necessary condition being (considering the last inequality) $e_{H L}^{V}>\frac{k}{\sqrt{\theta}}$. But note that $e_{H L}^{F B, V}=\frac{1}{\theta}$ and it has to be that $e_{H L}^{F B, V} \geq \frac{k}{\sqrt{\theta}}$ or else that $k \leq \frac{1}{\sqrt{\theta}}<1$. So the marginal productivity of labor in the nonvocational sector $k$ must be sufficiently low in order for $P C_{L L}^{V}$ to be omitted from the program (because it is implied by $P C_{H L}^{V}$ ); otherwise both $P C_{L L}^{V}$ and $P C_{H L}^{V}$ might be relevant.

Moreover, adding the incentive compatibility constraints two by two, the following implementability condition holds

$$
e_{L H}^{V}>\max \left\{e_{L L}^{V} ; e_{H H}^{V}\right\} \geq \min \left\{e_{L L}^{V} ; e_{H H}^{V}\right\}>e_{H L}^{V} .
$$

Concerning intermediate types, one has either $e_{L L}^{V}=e_{H H}^{V}$ or

$$
e_{H H}^{V}>e_{L L}^{V} \quad \text { and } \quad e_{L L}^{V}+e_{H H}^{V} \leq \frac{2 \gamma}{\Delta \theta}
$$

or

$$
e_{L L}^{V}>e_{H H}^{V} \quad \text { and } \quad e_{L L}^{V}+e_{H H}^{V} \geq \frac{2 \gamma}{\Delta \theta} .
$$

In words, if motivation has a higher impact on effort and output provision than ability (i.e. condition 6 is satisfied), then from the principal's viewpoint, types can be ordered as $L H \succ H H \succ L L \succ L H$. We call this instance motivation prevails (Case $M$ ). If, instead, ability has a higher impact on effort and output provision than motivation (i.e. condition 7 is satisfied), then from the principal's viewpoint, types can be ordered as $L H \succ L L \succ H H \succ L H$. We call this situation ability prevails (Case A). Finally, when neither ability nor motivation prevail, it becomes impossible for the principal to separate intermediate types and a pooling contract for types $H H$ and $L L$ is the solution to problem $P V F D$.

We omit here a detailed description of the equilibrium contracts that solve program $P V F D$ because it is beyond the scope of the present analysis. As said, optimal contracts can be found by extending the results in Barigozzi and Burani (2013) so as to allow for participation constraint $P C_{L L}^{V}$ to be binding (rather than considering $P C_{H L}^{V}$ only). ${ }^{14}$ We only state a result that parallels the one obtained in the preceding Section 4.1.

Proposition 2 The vocational principal is fully dominant if $k<1$.

When $k=1$, the mission-oriented principal is indifferent between hiring all types of workers or hiring only motivated types, and non-motivated types are indifferent between accepting the contracts offered by the non-vocational principal or the contracts offered by the vocational rival. We refer the reader to Section 5.3 (and to Situation (ii) with $P N V$ in Case 3, in particular) for a description of this alternative situation.

\footnotetext{
${ }^{14}$ The complete solution to program $P V F D$ is available upon request.
} 


\section{Competing principals}

Suppose that $1 \leq k \leq 1+\gamma$, whereby neither principal fully dominates and both principals truly compete for the exclusive services of the agent. The two principals simultaneously offer the worker the choice of a menu of screening contracts.

Now it is immediate to see that principal $V$ has an advantage in hiring motivated types $L H$ and $H H$ while principal $N V$ has an incentive in attracting non-motivated types $L L$ and $H L$. In other words, the mission-oriented principal is still dominant but only with respect to the set of motivated types, whereas the non-vocational principal is dominant with respect to the set of non-motivated types.

In equilibrium, we expect the principal, who is dominant relatively to a given set of types, to hire these types and to offer to the remaining types out-of-equilibrium contracts that will never be accepted.

\subsection{The non-vocational principal}

As for principal $N V$, it is again true that any incentive compatible contracts must be such that workers with the same skills are required to exert the same effort, $e_{i H}^{N V}=e_{i L}^{N V}=e_{i}^{N V}$, receive the same wage, $w_{i H}^{N V}=w_{i L}^{N V}=w_{i}^{N V}$ and enjoy the same utility $U_{i H}^{N V}=U_{i L}^{N V}=U_{i}^{N V}$, for $i=L, H$, irrespective of their (potential) intrinsic motivation.

Moreover, the non-vocational principal is dominated with respect to motivated workers, so he anticipates that in equilibrium he is going to attract non-motivated types only. Thus, he designs his contracts uniquely considering the outside options of non-motivated workers. In other words, in order to succeed in hiring non-motivated types $L L$ and $H L$, principal $N V$ must be able to leave them at least $U_{L L}^{V}$ and $U_{H L}^{V}$, respectively. Such reservation utilities are endogenous but, because of the simultaneity of moves, are taken as given by principal $N V$.

Then, the non-vocational principal's program is now

$$
\max _{\left(e_{i \cdot}^{N V}, w_{i}^{N V}\right)} E\left(\pi^{N V}\right)=\frac{1}{2}\left[\left(k e_{L}^{N V}-w_{L}^{N V}\right)+\left(k e_{H \cdot}^{N V}-w_{H}^{N V}\right)\right]
$$

subject to the two participation constraints of non-motivated types

$$
w_{i}^{N V}-\frac{1}{2} \theta_{i}\left(e_{i \cdot}^{N V}\right)^{2} \geq U_{i L}^{V}
$$

for every $i=L, H$ and two incentive compatibility constraints

$$
w_{i \cdot}^{N V}-\frac{1}{2} \theta_{i}\left(e_{i \cdot}^{N V}\right)^{2} \geq w_{i^{\prime} \cdot}^{N V}-\frac{1}{2} \theta_{i}\left(e_{i^{\prime} \cdot}^{N V}\right)^{2} \quad\left(I C_{i \cdot v s i^{\prime} \cdot}^{N V}\right)
$$

for every $i=L, H$ and $i^{\prime} \neq i$.

One can replicate the analysis which has been carried out in Section 4.1, substituting the total surplus utilities $U_{L H}^{T S, V}-U_{H H}^{T S, V}$ with $U_{L L}^{V}-U_{H L}^{V}$ and, accordingly, substituting the participation constraints 
$P C_{L H}^{N V}$ and $P C_{H H}^{N V}$ with $P C_{L L}^{N V}$ and $P C_{H L}^{N V}$, respectively. The five different regimes are still in place and so are the optimal effort levels associated with each regime. Figure 1 represents the reaction function of principal $N V$ who is interested in hiring non-motivated types only.

Insert Figure 1 around here

Notice that, when $P C_{i L}^{N V}$ is binding for the type with effort cost $i=L, H$, then it must necessarily be the case that $P C_{i L}^{V}$ is binding as well. In other words, $U_{i H}^{N V}=U_{i L}^{N V}=U_{i L}^{V}$ and type $i L$ is indifferent between working in either sector (the tie-breaking rule mentioned at the end of Section 2 might then apply). Conversely, when $P C_{i L}^{N V}$ is slack, then it must be the case that $U_{i L}^{N V}>U_{i L}^{V}$ and that type $i L$ strictly prefers to work in the standard sector rather than in the mission-oriented sector.

\subsection{The vocational principal}

Differently from the non-vocational principal, the mission-oriented principal offers four contracts when possible: worker $i j$ exerts effort $e_{i j}^{V}$, receives a wage $w_{i j}^{V}$ and enjoys utility $U_{i j}^{V}$. In equilibrium, principal $V$ will hire motivated agents only but will offer out-of-equilibrium contracts to non-motivated types so as to satisfy internal incentive compatibility.

In order to solve principal's $V$ program, we start by taking as given each one of the possible five regimes in which principal $N V$ can find himself. This allows to single out which participation constraint between $P C_{L L}^{N V}$ and $P C_{H L}^{N V}$ is binding. When $P C_{i L}^{N V}$, with $i=L, H$, is binding, it means that $P C_{i L}^{V}$ is binding as well and that type $i L$ is indifferent between the two principals. Then the dominated principal $V$ will offer this type her first best total surplus and make zero profits from that type. In fact, any other strategy would create the opportunity for profitable deviations by the competitor. In particular, if $P C_{L L}$ is binding, then $U_{L L}^{N V}=U_{L L}^{T S, V}=\frac{1}{2}$ and the mission-oriented firm obtains zero profit on the out-of-equilibrium contract for worker $L L$. In the same way, if $P C_{H L}$ is binding then $U_{H L}^{N V}=U_{H L}^{T S, V}=\frac{1}{2 \theta}$ and the mission-oriented principal earns zero profit from worker $H L .^{15}$

For the sake of concreteness, let us suppose that the non-vocational principal is in the situation where irrelevance of outside options holds (Case 1 in Figure 1). Then $P C_{L L}^{N V}$ is slack while $P C_{H L}^{N V}$ is binding. Thus, we can study a program for the mission-oriented principal which is similar to PVFD, but which is such that $P C_{L L}^{V}$ is slack and $P C_{H L}^{V}$ is binding, whereby the contract offered to type $H L$ satisfies $e_{H L}^{V}=e_{H L}^{F B, V}=\frac{1}{\theta}=w_{H L}^{V}$ and $U_{H L}^{V}=U_{H L}^{T S, V}=\frac{1}{2 \theta}$. The solution will clearly depend on whether motivation or ability prevails, that is on whether condition (6) or condition (7) holds or none. In case of

\footnotetext{
${ }^{15}$ Note that, because of competition, the mission-oriented principal reaches internal incentive compatibility at a higher cost than when he does not face any rival principal. In particular, by increasing (with respect to the monopsony case with zero outside options for all types) the utility that non-motivated workers obtain out of equilibrium, the vocational principal must pay larger information rents to motivated workers.
} 
multiple solutions, we take the one guaranteeing the highest profits to principal $V$. Once the bidimensional screening problem of the mission-oriented principal is solved, the utility $U_{L L}^{V}$ is also well defined so that the value $U_{L L}^{V}-U_{H L}^{T S, V}$ which enters the solution of program $P N V C$ is fully determined. The last step consists in checking whether the difference $U_{L L}^{V}-U_{H L}^{T S, V}$ that has been found solving the vocational principal's program is compatible with the bounds which define Case 1 for principal $N V$. If yes, then the solution obtained is an equilibrium, otherwise it must be discarded. We repeat the same procedure for all the other regimes for principal $N V$, from 2 to 5 .

Notably, the difference in reservation utilities $U_{L L}^{V}-U_{H L}^{V}$ is never too big so as to yield Cases 4 and 5. Therefore, countervailing incentives are never observed at equilibrium.

This analysis is relegated to Appendix C. ${ }^{16}$

\subsection{Sorting according to motivation}

In what follows, we characterize the optimal incentive schemes offered by the two competing principals when $1 \leq k \leq 1+\gamma$ and when workers optimally sort themselves by motivation, with motivated types $L H$ and $H H$ choosing to be employed in the mission-oriented sector and non-motivated types $L L$ and $H L$ being hired in the standard sector.

Different situations emerge according to the magnitude of $k$, which governs principal $N V$ 's regimes, and of $\gamma$ and $\theta$, that influence the states of the world for principal $V$. In what follows we refer to "second best" effort levels and contracts as the efforts and contracts that are designed by a monopsonist hiring workers with zero outside options.

Situation (i) When $k$ and $\gamma$ are both high, i.e. when $\bar{k}=\frac{(2 \theta-1)}{\theta} \leq k \leq 1+\gamma$ and $\bar{\gamma}=\frac{(\theta-1)}{\theta} \leq \gamma<1$, then the optimal incentive schemes are unique:

$P N V$ is always in Case 1 (irrelevance of outside options) and offers the second-best contracts with $e_{L H}^{N V}=e_{L L}^{N V}=k$ and $e_{H H}^{N V}=e_{H L}^{N V}=\frac{k}{(2 \theta-1)}$.

$P V$ motivation always prevails: employed types $L H$ and $H H$ are required to make efforts $e_{L H}^{F B, V}=$ $1+\gamma$ and $e_{H H}^{S B, V}=\frac{1+\gamma}{(2 \theta-1)}$, types $L L$ and $H L$ are offered out-of-equilibrium pooling contracts with effort $e_{L L}^{V}=e_{H L}^{V}=\frac{1}{\theta}$, respectively.

Situation (ii) When $k$ is such that $1 \leq k \leq \bar{k}$, then the optimal incentive schemes are not unique:

$P N V$ - when $1 \leq k<\underline{k}=\sqrt{\theta}$ principal $N V$ is in Case 3 and offers the first best contracts to all workers with efforts $e_{L H}^{F B, N V}=e_{L L}^{F B, N V}=k$ and $e_{H H}^{F B, N V}=e_{H L}^{F B, N V}=\frac{k}{\theta}$,

\footnotetext{
${ }^{16}$ There, it is shown that the difference in reservation utilities $U_{L L}^{V}-U_{H L}^{V}$ is never too big so as to yield Cases 4 and 5.
} 
- when $\underline{k} \leq k<\bar{k}$ principal $N V$ is in Case 2 and requires optimal effort levels $e_{L H}^{F B, N V}=$ $e_{L L}^{F B, N V}=k$ and $e_{H H}^{*, N V}=e_{H L}^{*, N V}=\frac{1}{\sqrt{\theta}}$.

$P V$ - when $0<\gamma<\gamma_{A}=(\theta-1)$, ability prevails (Case $A$ ), motivated types are asked to provide first-best effort levels $e_{L H}^{F B, V}=1+\gamma$ and $e_{H H}^{F B, V}=\frac{1+\gamma}{\theta}$ and non-motivated types are offered out-of-equilibrium contracts with first-best effort levels $e_{H L}^{F B, V}=\frac{1}{\theta}$ and $e_{L L}^{F B, V}=1$ and all the surplus.

- for $\gamma_{A} \leq \gamma \leq \gamma_{M}=2(\theta-1)$, neither ability nor motivation prevail, intermediate types' effort levels are pooled and the first-best total surplus is offered out-of-equilibrium to non-motivated types, whereby $e_{L H}^{F B, V}=1+\gamma, e_{H H}^{V}=e_{L L}^{F B, V}=1$ and $e_{H L}^{F B, V}=\frac{1}{\theta}$.

- for $\gamma_{M}<\gamma \leq 1$ and $\theta<\frac{3}{2}$ (ensuring that $\gamma_{M}<1$ ), motivation prevails (Case $M$ ), motivated types are required to provide effort levels $e_{L H}^{F B, V}=1+\gamma$ and $e_{H H}^{S B, V}=\frac{1+\gamma}{2 \theta-1}$ and non-motivated types are offered out-of-equilibrium the first-best total surplus, whereby $e_{H L}^{F B, V}=\frac{1}{\theta}$ and $e_{L L}^{F B, V}=1$.

As said, a more detailed discussion of the above results, is provided in Appendix C.

It can be checked that, given ability, motivated types who are all hired in the mission oriented sector provide higher effort than non-motivated types who are all hired in the standard sector, except for lowability types in Situation (ii) when motivation prevails for $P V$ and $P N V$ is in Case 3.

Figure 2 illustrates Situation (i) and Situation (ii) from the vocational principal's point of view, that is according to the relative magnitudes of $\gamma$ and $\theta$, the parameters representing the agent's private information.

Insert Figure 2 around here

The propositions that follows summarize what we have found so far.

Proposition 3 If $1 \leq k \leq 1+\gamma$, the sorting of workers types is efficient. All motivated types choose to work for the vocational principal and all non-motivated types choose to work for the non-vocational principal.

Corollary 1 Workers' self-selection is ability-neutral since average ability is identical across sectors.

The latter result would differ when the distribution of types is not uniform and independent: it is possible to verify that, if ability and intrinsic motivation are negatively correlated, then workers again self-select according to motivation but average ability is lower in the vocational sector relative to the non-vocational one.

As mentioned at the end of Section 4.1, when $k=1+\gamma$ the equilibrium is the one described in Situation (i) and it can be shown that the non-vocational principal does not have any incentive to deviate neither 
by trying to hire all types of workers, nor by trying to employ all types of workers except $H H$, nor by trying to employ all potential applicants except type $L H$.

We further propose a taxonomy of the above-mentioned equilibria with respect to the degree of competition between principals, which in turn depends on whether principals are sufficiently different in both technology and in the impact of the workers' motivation.

Proposition 4 In both sectors, effort provided by high-ability workers at equilibrium is not distorted. Moreover:

- If competition is mild, i.e. if both $k$ and $\gamma$ are high, then both principals ask low-ability hired workers to provide the second-best effort levels (see Situation (i)).

- If competition is harsh, i.e. if both $k$ and $\gamma$ are low, then both principals ask low-ability hired workers to provide first-best effort levels (see Situation (ii) with Case 3 for PNV and Case A for PV).

- Otherwise, i.e. if $k$ is high and $\gamma$ is low or vice-versa, then the principal who is relatively more efficient asks low-ability hired worker to provide an effort level which is in-between first- and secondbest, while the other principal requires first-best efforts.

The intuition behind these results is the following. When competition is mild, because principals are sufficiently differentiated from each other, then outside options are not particularly relevant and internal incentive compatibility is the driving force in determining equilibrium efforts for hired workers, which are the same as under monopsony. Conversely, when competition is harsh, because principals are similar to each other, then outside options are the determinant of equilibrium efforts for hired workers, while internal incentive compatibility plays a minor role. This outcome is the one that most resembles the full information equilibrium corresponding to Bertrand competition with each firm requiring first-best effort levels and offering wages such that the best offer of the competitor is met. Also note that this outcome is the one satisfying the so called separation property whereby competition among principals only affects the agents' compensation schemes but not the optimal effort levels (see Biglaiser and Mezzetti 2000, and the references therein). In-between these polar degrees of competition, there are instances in which one principal is relatively more efficient than the other (namely $P V$ when $\gamma$ is high and $k$ is low or $P N V$ when $k$ is high and $\gamma$ is low); for such a principal the separation property does not hold, since distortions in the agents' effort levels are reduced with respect to the second-best contracts. When these intermediate degrees of competition occur, then the less efficient principal asks for the first best effort levels in and out of equilibrium.

We can conclude that distortions in effort provision are always decreasing with the level of competition and, in case of intermediate degrees of competition, only the principal who is relatively more efficient implements screening contracts in equilibrium. 
Finally, it is worth mentioning that competition between principals never leads to countervailing incentives, namely upward distortions in effort levels never occur.

\subsection{Market segmentation, wage differentials and the power of incentives}

In this section we compare the wage schemes offered in the two sectors of the labor market. In particular, it is interesting to consider the model's predictions as for the wage differential, if any, between the vocational sector and the standard one. Thus, we first compare the wage rate offered by the two principals to motivated and non-motivated workers, fixing the level of ability. Then, we consider the return to ability to verify whether and under which conditions the mission-oriented sector is characterized by lower power of incentives, as some authors suggested (see, as an example, Handy and Katz 1998 and Besley and Gathak 2005). All results in this section are derived in Appendix D.

For a wide range of parameter configurations, it can be shown that

$$
w_{L H}^{V}<w_{L H}^{N V}=w_{L L}^{N V}
$$

and also that

$$
w_{H H}^{V}<w_{H H}^{N V}=w_{H L}^{N V}
$$

This result holds when Situation $(i)$ occurs and $k$ is sufficiently high, namely when competition is mild, or when Situation (ii) holds, ability prevails for the vocational principal (meaning that $\gamma$ must be low) and $k$ is still sufficiently high, namely when the non-vocational principal is relatively more efficient. We refer the reader to Appendix D for the detailed analysis of all possible instances.

The intuition for this result is the following: both when Situation $(i)$ holds and when ability prevails and $\gamma$ is low, then internal incentive compatibility for the vocational principal is such that motivated agents do not cumulate large information rents. These agents are thus offered low wages. This fact depressed the left-hand side of the above inequalities. On the other hand, when $k$ is high, principals are sufficiently differentiated and this raises the wages that are paid in the standard sector, thus raising the right-hand side of the inequalities above.

So we indeed observe a compensating wage differential across the two sectors which is entirely driven by the intrinsic motivation and does not depend on the differences in workers' ability. Nonetheless, ability does matter in that inequality (8) is easier to be satisfied than inequality (9). In other words, it might be the case that a compensating wage differential exists for high ability types but not for low ability workers or that the wage differential experienced by high ability workers be larger than the wage gap existing for low-skilled workers. This supports the empirical findings that the compensating wage differential is increasing in ability and that the wage penalty across sectors is more severe at the top of the wage ladder rather than at the bottom. The fact that the public sector wage penalty is higher for managers and top 
executives with respect to lower levels in the hierarchy is documented by Roomking and Weisbrod (1999) and Bargain and Melly (2008) among others. ${ }^{17}$

Finally, note that when the wage differential is in place, it is always the case that $e_{L H}^{V}>e_{L H}^{N V}=e_{L L}^{N V}$ and $e_{H H}^{V}>e_{H H}^{N V}=e_{H L}^{N V}$. Hence, effort provision is higher in the vocational sector where wage rates are lower.

Let us consider now the difference between return to ability in the mission-oriented $\left(w_{L H}^{V}-w_{H H}^{V}\right)$ and in the standard sector $\left(w_{L L}^{N V}-w_{H L}^{N V}\right)$. In particular, if

$$
w_{L H}^{V}-w_{H H}^{V}<w_{L L}^{N V}-w_{H L}^{N V}
$$

then the power of incentives is lower in the mission-oriented sector because, in equilibrium, the gain from increased ability is lower in such a sector.

By analyzing wages in the different equilibria one can check that the mission-oriented sector is characterized by low power of incentives in a superset of the region of parameters where the wage differential exists. In particular, it realizes in the same situations where workers exert a higher effort in the missionoriented than in the standard sector. Conversely, the mission-oriented sector never displays lower power of incentives when the degree of competition is intermediate and principal $V$ is relatively more efficient.

In the end, a lower power of incentives in the mission oriented market is compatible both with a high and with a low level of competition between the two principals.

The remark that follows fixes the main ideas illustrated in this section.

Proposition 5 When the standard sector is relatively more efficient ( $k$ is high), (i) a compensating wage differential occurs and motivated workers receive a lower salary in exchange for a higher level of effort provision with respect to non-motivated workers; (ii) in the mission-oriented sector, the power of incentives is lower than in the standard sector.

Result (i) suggests that, when the competitor is characterized by an efficient technology, it might be particularly worth for a firm to differentiate itself according to the choice of a mission. Indeed, our framework could be extended to allow principals to endogenously determine their missions, in line with Besley and Ghatak (2005).

\section{Conclusion}

Some recent papers on intrinsic motivation (Besley and Ghatak 2005, Delfgaauw and Dur 2008 and 2010) analyze the problem of sorting of workers with different characteristics, being motivation one of

\footnotetext{
${ }^{17}$ We refer the reader to the excellent review of the literature contained in Delfgaauw and Dur (2010).
} 
them, to different firms/sectors where jobs vary in intrinsic qualities. Typically, two or more competing firms/sectors are considered: in some of them workers care about the organizational mission or intrinsically value their personal contribution to the firm's outcome and in another employees only care about extrinsic rewards.

We contribute to this literature by analyzing a framework were each sector is represented by a firm interested in the exclusive services of a worker. The latter has private information on her skills and on her motivation to contribute to the firms' outcome. Firms differ both in their production technology and in the mission they pursue and a motivated worker is keen to be hired by the mission-oriented firm. From a technical point of view, we characterized the optimal contracts offered by two competing principals in the presence of bidimensional adverse selection, where contracts are characterized by an effort level and a wage rate.

Our aim is to study the sorting of worker's types into the different sectors and to make predictions relative to the average motivation and average ability of workers in each sector. In particular we are interested in assessing whether workers with low ability tend to be hired in the mission-oriented sector and thus whether a selection bias might cause a wage penalty for motivated workers employed by the mission-oriented firm.

We find that, when the firms' technology is not too different so that no firm strictly dominates the other one, then the market is fully segmented: agents sort themselves by motivation, with motivated types being employed by the mission-oriented principal and non-motivated types being hired by the standard principal.

We also show that a wage penalty in the mission-oriented sector can be observed when the degree of competition is not too high and the standard firm is relatively more efficient. Such a wage gap is not caused by a selection bias because workers sort themselves along the dimension of motivation while the most able workers are evenly distributed across sectors. When motivated workers suffer a wage penalty, our model also predicts higher effort levels and lower power of incentives in the mission oriented sector.

In general, equilibrium contracts are defined by the tension between competition, leading to first-best effort levels, and information rents extraction that drives to downward effort distortions. As a result effort distortions are decreasing in the degree of competition between the two firms.

Our results extend the findings of Delfgaauw and Dur (2010) to the context of asymmetric information about both worker's characteristics, that is intrinsic motivation and ability and to the case of strategic interaction between the firms/principals representing the two sectors of the labor market. Under complete information and perfect competition, Delfgaauw and Dur (2010) show that, when intrinsic motivation is output-oriented, that is when intrinsic motivation depends on effort or on one's personal contribution to output, then selection into the public sector is ability-neutral. Such a result is confirmed in our setting. We also add to Delfgaauw and Dur (2008), where asymmetric information on both workers' motivation 
and laziness is considered. In their framework the standard sector is perfectly competitive and thus non-strategic and the mission-oriented sector is cost-minimizing and interested in hiring only two worker types at most. Moreover, their setting does not allow the comparison of average productivity between the two sectors.

\section{A Appendix}

\section{B Competition under full information}

Wages offered by the two principals in equilibrium are:

$$
\begin{aligned}
& w_{L H}^{* V}=\underbrace{\frac{k^{2}}{2}}_{\text {outside option }}+\underbrace{\frac{1}{2}(1+\gamma)^{2}-\gamma(1+\gamma)}_{\text {net cost of effort }} \\
& w_{H H}^{* V}=\underbrace{\frac{k^{2}}{2 \theta}}_{\text {outside option }}+\underbrace{\frac{1}{2} \frac{(1+\gamma)^{2}}{\theta}-\gamma \frac{(1+\gamma)}{\theta}}_{\text {net cost of effort }} \\
& w_{L L}^{* N V}=\underbrace{\frac{1}{2}}_{\text {outside option }}+\underbrace{\frac{1}{2} k^{2}}_{\text {cost of effort }} \\
& w_{H L}^{* N V}=\underbrace{\frac{1}{2 \theta}}_{\text {outside option }}+\underbrace{\frac{1}{2 \theta} k^{2}}_{\text {cost of effort }}
\end{aligned}
$$

Where the first part in the expression covers the outside option (the best offer of the competitor) while the second part rewards the (net) cost of the first best effort.

Note that outside options do not depend on intrinsic motivation.

We can summarize the equilibrium under full information as follows.

Remark 2 Competition under full information when $1<k<1+\gamma$. The market is fully segmented: principal $V$ hires motivated workers while principal NV hires non-motivated ones. All effort levels are at the first best and wages are the ones described in (10). Both principals earn positive profits on the types they hire and workers receive a positive utility corresponding to the best offer that the less productive firm is able to make.

In the case of $k=1$ and $k=1+\gamma$ principals are equally productive in hiring non-motivated and motivated types respectively. The tie is broken in favor of the more productive principal. Thus, when $k=1$ principal $V$ is hiring all the workers and is earning positive profits only on motivated ones. When $k=1+\gamma$ principal $N V$ is hiring all the workers and is earning positive profits only on non-motivated ones. 


\section{Optimal contracts with competing principals}

As mentioned in the main text, when $1 \leq k \leq 1+\gamma$ and none of the principals is fully dominant, we proceed by taking one of the regimes in which principal $N V$ might find himself (starting from Case 1 and moving to Case 5 ) as given. We then solve for the vocational principal's optimal incentive schemes and finally check whether the value of $U_{L L}^{V}-U_{H L}^{V}$ thus obtained is compatible with the selected case for principal $N V$.

\section{C.1 $P N V$ is in Case 1}

When the non-vocational principal is in Case 1, the only binding participation constraint is $P C_{H L}^{N V}$. Therefore, type $H L$ must be indifferent between the two sectors and $P C_{H L}^{V}$ must be binding as well. The vocational principal offers to this type the first-best effort level and makes zero profits from this type of agent, whereby $e_{H L}^{V}=\frac{1}{\theta}$ and $U_{H L}^{T S, V}=\frac{1}{2 \theta}$.

\section{C.1.1 Motivation prevails}

Suppose further that motivation prevails for the vocational principal, whereby optimal effort levels must be ordered as $e_{L H}>e_{H H}>e_{L L} \geq e_{H L} \cdot{ }^{18}$

Full separation of types One could solve a problem in which each type of worker gets a different contract and in which the binding constraints are the downward incentive compatibility ones $I C_{L H v s H H}$, $I C_{H H v s L L}$ and $I C_{L L v s H L}$ together with $P C_{H L}$. Solving the binding constraints for the wage rates, substituting them into the principal's objective function and maximizing it with respect to effort levels (omitting $e_{H L}$ which is already determined) yields

$$
e_{L H}=1+\gamma \quad e_{H H}=\frac{1+\gamma}{2 \theta-1} \quad e_{L L}=\frac{1-2 \gamma}{3-2 \theta} \quad e_{H L}=\frac{1}{\theta}
$$

This candidate solution with full separation of types exists for $\theta<\frac{3}{2}$ and $\frac{4(\theta-1)}{(2 \theta+1)}=\underline{\gamma}^{M}<\gamma<\bar{\gamma}^{M}=\frac{3(\theta-1)}{2 \theta}$, where inequalities $\underline{\gamma}^{M}<\gamma$ and $\gamma<\bar{\gamma}^{M}$, respectively, are equivalent to the monotonicity conditions $e_{H H}>e_{L L}$ and $e_{L L}>e_{H L} \cdot{ }^{19}$ Profits to the vocational principal from hired types $L H$ and $H H$ are equal to

$$
\pi^{M, F S}=\frac{1}{4}\left(\frac{\theta(1+\gamma)^{2}}{(2 \theta-1)}+\frac{(1-2 \gamma)(\theta-4 \gamma+2 \theta \gamma-1)}{(3-2 \theta)^{2}}-\frac{(2 \theta-1)}{\theta^{2}}\right)
$$

There remains to compute the outside option left by Principal $V$ to type $L L$, which is given by $U_{L L}^{V}=$ $w_{L L}^{V}-\frac{1}{2} e_{L L}^{2} ;$ substituting for $w_{L L}^{V}=\frac{1}{2} e_{L L}^{2}+\frac{(2 \theta-1)}{2 \theta^{2}}$ (which has been found imposing $I C_{L L v s H L}$ to bind)

\footnotetext{
${ }^{18}$ From now on, when no confusion arises, we omit the superindex relative to the type of principal considered.

${ }^{19}$ All omitted participation and incentive compatibility constraints have been checked to hold ex-post. The same is true for all subsequent problems but we avoid to repeat a similar statement each time.
} 
yields $U_{L L}^{V}=\frac{(2 \theta-1)}{2 \theta^{2}}$ and thus $U_{L L}^{V}-U_{H L}^{V}=\frac{(\theta-1)}{2 \theta^{2}}$. Such difference in reservation utilities is compatible with $P N V$ being in Case 1 if and only if $\frac{(\theta-1)}{2 \theta^{2}}<\frac{k^{2}(\theta-1)}{2(2 \theta-1)^{2}}$ or else if and only if

$$
k>\frac{(2 \theta-1)}{\theta}=\bar{k}
$$

where $\bar{k}>1$ always holds while $\bar{k}<1+\gamma$ iff

$$
\gamma>\frac{\theta-1}{\theta}=\bar{\gamma}
$$

Note that $\bar{\gamma}<\underline{\gamma}^{M}$ always holds, so the condition $\gamma>\bar{\gamma}$ is always verified when motivation prevails, and $\bar{k}<1+\gamma$ is true in this case.

Pooling between non-motivated types $L L$ and $H L$ Suppose that $P C_{H L}^{V}$ is till binding but that a pooling contract is offered to the non-motivated types whereby optimal effort levels are ordered as $e_{L H}>e_{H H}>e_{L L}=e_{H L}=\frac{1}{\theta}$, and wages are such that $w_{H L}=w_{L L}=\frac{1}{\theta}$ (again, $P V$ makes zero profits on types that he is not able to hire). Optimal effort levels are given by

$$
e_{L H}=1+\gamma \quad e_{H H}=\frac{1+\gamma}{2 \theta-1} \quad e_{L L}=e_{H L}=\frac{1}{\theta}
$$

This solution exists when the monotonicity condition $e_{H H}>e_{L L}$ is satisfied which is equivalent to $\gamma>\frac{\theta-1}{\theta}=\bar{\gamma}$, and it holds for a superset of parameter configurations relative to the fully separating solution when motivation prevails. Profits for $P V$ from the hired types $L H$ and $H H$ are given by

$$
\pi^{M, \text { Pool }_{L L+H L}}=\frac{1}{8}\left((1+\gamma)^{2}+\frac{(1+\gamma)^{2}}{(2 \theta-1)}-\frac{2(2 \gamma+1)}{\theta}\right)
$$

and it can be checked that $\pi^{M, \text { Pool }_{L L+H L}}>\pi^{M, F S}$ iff $\gamma>\frac{(\theta-1)(3-\theta)}{2 \theta(2-\theta)}=\gamma_{1}$ where $\gamma_{1}<\underline{\gamma}^{M}$ always holds for $\theta<\frac{3}{2}$. Hence when motivation prevails and both solutions with full separation and pooling between non-motivated types are in place, then pooling is strictly preferred by $P V$ to full separation and thus the latter solution can be discarded. Finally, outside options for non-motivated types are the same as in the previous case, whereby $U_{L L}^{V}-U_{H L}^{V}=\frac{(\theta-1)}{2 \theta^{2}}$ and compatibility with Case 1 for $P N V$ is still given by the condition $k>\bar{k}$.

\section{C.1.2 Pooling between intermediate types}

Suppose now that the optimal effort levels offered by $P V$ are ordered as $e_{L H}>e_{H H}=e_{L L}>e_{H L}$. There are two possible types of solutions with pooling of intermediate types, depending on whether $I C_{H H v s H L}$ or $I C_{L L v s H L}$ binds first.

Case (a) Suppose that $I C_{H H v s H L}$ is binding while $I C_{L L v s H L}$ is slack: we call this situation Case (a) and denote it with the superscript $P a$. Consider further $P C_{H L}$ and $I C_{L H v s H H}$ as binding constraints so 
that optimal effort levels are given by

$$
e_{L H}=1+\gamma \quad e_{H H}=e_{L L}=\frac{1+\gamma}{2 \theta-1} \quad e_{H L}=\frac{1}{\theta}
$$

Monotonicity condition $e_{H H}=e_{L L}>e_{H L}$ holds iff $\gamma>\bar{\gamma}$ and $I C_{H H v s H L}$ is binding while $I C_{L L v s H L}$ is slack iff $e_{H H}=e_{L L}+e_{H L}>\frac{2 \gamma}{\theta-1}$ or else iff $\gamma<\bar{\gamma}$. Since these two conditions are incompatible, Case $(a)$ can be discarded.

Case (b) Suppose now that $I C_{L L v s H L}$ is binding while $I C_{H H v s H L}$ is slack: we call this situation Case (b) and denote it with the superscript $P b$. Consider further $P C_{H L}$ and $I C_{L H v s L L}$ as binding constraints so that optimal effort levels are given by

$$
e_{L H}=1+\gamma \quad e_{H H}=e_{L L}=\frac{2-\gamma}{2} \quad e_{H L}=\frac{1}{\theta}
$$

Monotonicity condition $e_{H H}=e_{L L}>e_{H L}$ holds iff $\gamma<\frac{2(\theta-1)}{\theta}=\bar{\gamma}^{P b}=2 \bar{\gamma}$. Moreover, IC $C_{L L v s L}$ is binding while $I C_{H H v s H L}$ is slack iff $e_{H H}=e_{L L}+e_{H L}<\frac{2 \gamma}{\theta-1}$ or else iff $\gamma>\frac{2(\theta-1)(\theta+1)}{\theta(\theta+3)}=\underline{\gamma}^{P b}$. Hence Case $(b)$ exists iff $\underline{\gamma}^{P b}<\gamma<\bar{\gamma}^{P b}$. Since $\underline{\gamma}^{P b}>\bar{\gamma}$, Case $(b)$ coexists with the solution that is in place when motivation prevails and there is pooling between non-motivated types. Profits to $P V$ when hiring motivated types are given by

$$
\pi^{P b}=\frac{1}{8}\left((1+\gamma)^{2}+\frac{(2-3 \gamma)(2-\gamma)}{4}-\frac{2(2 \theta-1)}{\theta^{2}}\right)
$$

and it possible to show that $\pi^{P b}<\pi^{M, P_{0 o l} L+H L}$ whenever the two solutions coexist. So Case $(b)$ can be discarded.

\section{C.1.3 Ability prevails}

Suppose now that ability prevails for the vocational principal, whereby the solution to the vocational principal's program must be such that optimal effort levels are ordered as $e_{L H}>e_{L L}>e_{H H} \geq e_{H L}$. Here we follow Barigozzi and Burani (2013) and distinguish between two possible solutions with full separation of types: Case $A$.a that holds when $I C_{L H v s L L}, I C_{L L v s H H}$ and $I C_{H H v s H L}$ are binding or else when $e_{H H}+e_{H L}>\frac{2 \gamma}{\theta-1}$ and Case $A . b$ that holds when $I C_{L H v s L L}, I C_{L L v s H L}$ and $I C_{H H v s L L}$ are binding, or else when $e_{L L}+e_{H L}<\frac{2 \gamma}{\theta-1}<e_{L L}+e_{H H} .^{20}$

Case A.a In Case A.a the binding constraints are the downward incentive compatibility constraints $I C_{L H v s L L}, I C_{L L v s H H}$ and $I C_{H H v s H L}$ together with participation constraint $P C_{H L}$. In order for type

\footnotetext{
${ }^{20}$ Case $A . a$ corresponds to Case $A .1$ and Case $A . b$ corresponds to Case $A .3$ in the companion paper. When the distribution of types is not uniform another case emerges, called Case $A .2$, which is such that the binding constraints are $I C_{L H} v_{L} L$, $I C_{L L v H L}$ and $I C_{H H v s H L}$ and which holds for $e_{H H}+e_{H L}<\frac{2 \gamma}{\theta-1}<e_{L L}+e_{H L}$.
} 
$L L$ to obtain a non-negative information rent from mimicking type $H H$ it must also be the case that $e_{H H} \geq \frac{2 \gamma}{\theta-1}$, which is a more restrictive requirement than $e_{H H}+e_{H L}>\frac{2 \gamma}{\theta-1}$. Optimal effort levels are given by

$$
e_{L H}=1+\gamma \quad e_{L L}=1-\gamma \quad e_{H H}=\frac{(1+3 \gamma)}{3 \theta-2} \quad e_{H L}=\frac{1}{\theta} .
$$

Monotonicity condition $e_{L L}>e_{H H}$ holds iff $\gamma<\frac{3(\theta-1)}{(3 \theta+1)}=\bar{\gamma}_{1}^{A a}$ while $e_{H H}>e_{H L}$ holds iff $\gamma>\frac{2(\theta-1)}{3 \theta}=$ $\underline{\gamma}^{A a}$. Moreover the requirement $e_{H H} \geq \frac{2 \gamma}{\theta-1}$ is satisfied iff $\gamma \leq \frac{(\theta-1)}{3 \theta-1}=\bar{\gamma}_{2}^{A a}$, but $\bar{\gamma}_{2}^{A a}<\underline{\gamma}^{A a}$ and so this candidate solution can be discarded.

Case $A . b$ In Case $A . b$ the binding incentive compatibility constraints are $I C_{L H v s L L}, I C_{L L v s H L}$ and (upward) $I C_{H H v s L L}$ together with participation constraint $P C_{H L}$. Optimal effort levels are given by

$$
e_{L H}=1+\gamma \quad e_{L L}=\frac{(1-2 \gamma)}{(2-\theta)} \quad e_{H H}=\frac{(1+\gamma)}{\theta}=e_{H H}^{F B} \quad e_{H L}=\frac{1}{\theta}
$$

Monotonicity condition $e_{L L}>e_{H H}$ is satisfied iff $\gamma<\frac{2(\theta-1)}{(\theta+2)}=\bar{\gamma}^{A b}$ while condition $e_{L L}+e_{H L}<\frac{2 \gamma}{\theta-1}$ holds iff $\gamma>\frac{(\theta-1)}{\theta}=\bar{\gamma}$ where $\bar{\gamma}^{A b}<\bar{\gamma}$. So the above conditions are not compatible and Case $A . b$ can be discarded.

Pooling between motivated types Suppose now that a pooling contract is offered by $P V$ to motivated types whereby effort levels are ordered as $e_{L H}>e_{L L}>e_{H H}=e_{H L}=\frac{1}{\theta}$. The incentive compatibility constraints that one assumes to be binding are $I C_{L H v s L L}, I C_{L L v s H H}$ together with participation constraint $P C_{H L}$. Optimal effort levels are

$$
e_{L H}=1+\gamma \quad e_{L L}=1-\gamma \quad e_{H H}=e_{H L}=\frac{1}{\theta}
$$

This solution exists iff $\gamma<\bar{\gamma}$ or else iff the monotonicity condition $e_{L L}>e_{H H}$ holds. Reservation utilities are such that $U_{L L}^{V}-U_{H L}^{V}=\frac{(2 \theta-1)}{2 \theta^{2}}-\frac{1}{2 \theta}=\frac{(\theta-1)}{2 \theta^{2}}$ as in the previous regimes and compatibility with $P N V$ being in Case 1 occurs for $k>\frac{(2 \theta-1)}{\theta}=\bar{k}$. But note that $\bar{k}>1+\gamma$ holds whenever $\gamma<\bar{\gamma}$ so that the condition $k>\bar{k}$ can never be satisfied in this case and this candidate solution must be discarded.

\section{C.2 $P N V$ is in Cases from 2 to 4}

When the non-vocational principal is in Cases from 2 to 4 , the binding participation constraints are both $P C_{H L}^{N V}$ and $P C_{L L}^{N V}$. Therefore, both $P C_{H L}^{V}$ and $P C_{L L}^{V}$ must be binding as well and both types $H L$ and $L L$ must be indifferent between the two sectors. The vocational principal offers them first-best effort levels and makes zero profits from these types of agent, whereby $e_{H L}^{V}=\frac{1}{\theta}$ and $U_{H L}^{T S, V}=\frac{1}{2 \theta}$ together with $e_{L L}^{V}=1$ and $U_{L L}^{T S, V}=\frac{1}{2}$. Now the difference in reservation utilities for non-motivated types is fully determined and is equal to $U_{L L}^{T S, V}-U_{H L}^{T S, V}=\frac{1}{2}-\frac{1}{2 \theta}=\frac{(\theta-1)}{2 \theta}$. 


\section{C.2.1 Motivation prevails}

Suppose that motivation prevails for the vocational principal, whereby optimal effort levels must be ordered as $e_{L H}>e_{H H}>e_{L L}=1>e_{H L}=\frac{1}{\theta}$. The binding constraints are the downward incentive compatibility ones $I C_{L H v s H H}, I C_{H H v s L L}$ together with $P C_{L L}$ and $P C_{H L}$. Solving for the wage rates, substituting them into the principal's objective function and maximizing with respect to effort levels (omitting $e_{L L}$ and $e_{H L}$ which are already determined) yields

$$
e_{L H}=1+\gamma \quad e_{H H}=\frac{1+\gamma}{2 \theta-1} \quad e_{L L}=1 \quad e_{H L}=\frac{1}{\theta} .
$$

This candidate solution exists for $\theta<\frac{3}{2}$ and $\gamma<\gamma_{M}=2(\theta-1)$, where inequality $\gamma<\gamma_{M}$ is equivalent to the monotonicity condition $e_{H H}>e_{L L}$ and where $\gamma_{M}<1$ whenever $\theta<\frac{3}{2}$. Finally, profits to the vocational principal from hired types $L H$ and $H H$ are equal to

$$
\pi_{M}=\frac{\theta(1+\gamma)^{2}-(2 \gamma-\theta+2)(2 \theta-1)}{4(2 \theta-1)} .
$$

The difference in reservation utilities $U_{L L}^{T S, V}-U_{H L}^{T S, V}=\frac{(\theta-1)}{2 \theta}$ is compatible with $P N V$ being in Case 2 if and only if $\frac{k^{2}(\theta-1)}{2(2 \theta-1)^{2}}<U_{L L}^{V}-U_{H L}^{V}=\frac{(\theta-1)}{2 \theta} \leq \frac{k^{2}(\theta-1)}{2 \theta^{2}}$. As for the lower bound, it is satisfied when

$$
k<\frac{(2 \theta-1)}{\sqrt{\theta}}=\overline{\bar{k}}
$$

where $\overline{\bar{k}}>1$ always holds and $\overline{\bar{k}}<1+\gamma$ is true iff

$$
\gamma>\frac{(2 \theta-1)-\sqrt{\theta}}{\sqrt{\theta}}=\overline{\bar{\gamma}}
$$

where $\overline{\bar{\gamma}}<\gamma_{M}$. So $\overline{\bar{k}}$ is always included in the interval $(1 ; 1+\gamma)$ when motivation prevails. As for the upper bound, it is satisfied iff

$$
k \geq \sqrt{\theta}=\underline{k}
$$

with $\underline{k}>1$ and $\underline{k}<1+\gamma$ iff

$$
\gamma>\sqrt{\theta}-1=\underline{\gamma}
$$

where $\underline{\gamma}<\gamma_{M}$. Hence, $\bar{k}$ is also included in the interval $(1 ; 1+\gamma)$ when motivation prevails. Finally note that

$$
\underline{k}<\bar{k}<\overline{\bar{k}}
$$

always holds.

Conversely, $P N V$ is in Case 3 for $\frac{k^{2}(\theta-1)}{2 \theta^{2}}<U_{L L}^{V}-U_{H L}^{V}=\frac{(\theta-1)}{2 \theta} \leq \frac{k^{2}(\theta-1)}{2}$. The lower bound is satisfied for $k<\underline{k}$ while the upper bound holds iff

$$
k \geq \frac{1}{\sqrt{\theta}}=k_{4}
$$

where $k_{4}<1$ always holds. So $k \geq k_{4}$ is always satisfied and Case 4 cannot be compatible with motivation prevailing for $P V$. 


\section{C.2.2 Pooling between intermediate types}

Suppose that the ordering of optimal effort levels is such that $e_{L H}>e_{H H}=e_{L L}=1>e_{H L}=\frac{1}{\theta}$. Now the binding constraints are $I C_{L H v s L L}, P C_{L L}$ and $P C_{H L}$. Optimal effort levels are

$$
e_{L H}=1+\gamma \quad e_{H H}=e_{L L}=1 \quad e_{H L}=\frac{1}{\theta}
$$

and this solution exists iff

$$
\gamma \geq \frac{\theta-1}{2}=\gamma_{P}
$$

with $\gamma_{P}<\gamma_{M}$, which ensures that $I C_{H H v s H L}$ is satisfied. Note that at this solution $P V$ is making positive profits from type $L H$ only, which are equal to

$$
\pi_{P}=\frac{\gamma^{2}}{8}
$$

and which are always smaller than the profits when motivation prevails. So this solution only holds for $\gamma_{P} \leq \gamma<\gamma_{M}$.

This solution is compatible with Case 2 for $P N V$ iff $\underline{k}<k \leq \overline{\bar{k}}$, where $\overline{\bar{k}}<1+\gamma$ when $\gamma>\overline{\bar{\gamma}}$, with $\overline{\bar{\gamma}}>\gamma_{P}$. Hence when $\gamma_{P}<\gamma<\overline{\bar{\gamma}}$ we have $\overline{\bar{k}}>1+\gamma$ so the condition $k \leq \overline{\bar{k}}$ is always satisfied. The solution is also compatible with Case 3 holding for $P N V$ when $k \geq \underline{k}$, where $\underline{k}<1+\gamma$ iff $\gamma>\underline{\gamma}$ and $\underline{\gamma}<\gamma_{P}$. Thus, $\underline{k}<1+\gamma$ is always true when $\gamma_{P} \leq \gamma<\gamma_{M}$ and the pooling solution holds. Conversely, Case 4 can be neglected because the difference in reservation utilities is incompatible with values $k \geq 1$.

\section{C.2.3 Ability prevails}

Suppose now that ability prevails for $P V$ and that the ordering of optimal effort levels is such that $e_{L H}>e_{L L}=1>e_{H H}>e_{H L}=\frac{1}{\theta}$. Again one has to distinguish between Case A.a and Case A.b

Case A.a In Case A.a the binding incentive compatibility constraints are $I C_{L H v s L L}$ and $I C_{H H v s H L}$ together with participation constraints $P C_{L L}$ and $P C_{H L}$. Optimal effort levels are given by

$$
e_{L H}=1+\gamma \quad e_{L L}=1 \quad e_{H H}=\frac{(1+\gamma)}{\theta}=e_{H H}^{F B} \quad e_{H L}=\frac{1}{\theta}
$$

The monotonicity condition $e_{L L}>e_{H H}$ holds when $\gamma<(\theta-1)=\gamma_{A}$. This solution exists when $I C_{H H v s H L}$ binds before $I C_{H H v s L L}$, which occurs when $0<\gamma<\frac{(\theta-1)}{2}=\gamma_{P}<\gamma_{A}$. Compatibility conditions are the same as before, namely this solution is compatible with $P N V$ being in Case 3 for $1 \leq k \leq \underline{k}$ or in Case 2 for $\underline{k}<k \leq \overline{\bar{k}}$. But note that $\underline{k}<1+\gamma$ iff $\gamma>\underline{\gamma}$ and $\underline{\gamma}<\gamma_{P}$, then this solution is only compatible with $P N V$ being in in Case 3 when $0<\gamma \leq \underline{\gamma}$. Conversely, when $\underline{\gamma}<\gamma<\gamma_{P}$, this solution is compatible with $P N V$ being in Case 3 for $1 \leq k \leq \underline{k}$ or with $P N V$ being in Case 2 for $\underline{k}<k \leq 1+\gamma$, given that $\overline{\bar{k}}>1+\gamma$ when $\gamma<\gamma_{P}$. 
Case $A . b$ In Case $A . b$ the binding incentive compatibility constraints are $I C_{L H v s L L}$ and $I C_{H H v s L L}$ together with participation constraints $P C_{L L}$ and $P C_{H L}$. Optimal effort levels are the same as in (16) and this solution exists for $\gamma_{P} \leq \gamma<\gamma_{A}$. Within these bounds, the monotonicity condition $e_{L L}>e_{H H}$ is satisfied and $I C_{H H v s L L}$ binds before $I C_{H H v s H L}$. This solution coexists with pooling between intermediate types, therefore a comparison between profits associated with the two solutions is called for. Profits in this case are given by

$$
\pi_{A b}=\frac{1}{8}\left((1+\gamma)^{2}+\frac{(1+\gamma)^{2}}{\theta}-(4 \gamma+3-\theta)\right)
$$

and they are always higher than profits given by expression (15). Therefore Case A.b is chosen for $\gamma_{P} \leq \gamma<\gamma_{A}$, while pooling between intermediate types will be the solution only when $\gamma_{A} \leq \gamma \leq \gamma_{M}$. Compatibility of this solution with Case 3 for $P N V$ is ensured when $1 \leq k \leq \underline{k}$ and with Case 2 when $\underline{k}<k \leq 1+\gamma$, being $\overline{\bar{\gamma}}>\gamma^{*}$. Again Case 4 can be discarded.

Before turning to Case 5 for the non-vocational principal, straightforward computations lead us to observe that profits which principal $V$ makes when offering a pooling contract to non-motivated types and when $P N V$ is in Case 1 are always strictly higher than profits accruing to principal $V$ given that the rival principal $N V$ is in Cases 2-4. In other words, profits given by expression (12) are always strictly higher than those in expressions (13), (15) and (17). ${ }^{21}$ Hence the following result holds.

Result 1 The vocational principal always prefers the pooling contract offered to non-motivated types (described in C.1.1), given that the non-vocational principal is in Case 1, to any other optimal contract that he proposes when the non-vocational principal is in Cases 2-4.

\section{C.3 $P N V$ is in Case 5}

When the non-vocational principal is in Case 5, the only binding participation constraint is $P C_{L L}^{N V}$. Type $L L$ is indifferent between the two sectors and $P C_{L L}^{V}$ must be binding as well. The vocational principal offers the first-best effort level and makes zero profits from type $L L$, whereby $e_{L L}^{V}=1$ and $U_{L L}^{T S, V}=\frac{1}{2}$. Conversely, type $H L$ strictly prefers the non-vocational principal and is such that $U_{H L}^{N V}>U_{H L}^{V}$.

\section{C.3.1 Motivation prevails}

Suppose that motivation prevails for the vocational principal, whereby optimal effort levels are ordered as $e_{L H}>e_{H H}>e_{L L}=1 \geq e_{H L}$.

Full separation of types Assume that each type of agent is offered a different contract and that the binding constraints are the downward incentive compatibility ones $I C_{L H v s H H}, I C_{H H v s L L}$, the upward

\footnotetext{
${ }^{21}$ Profits associated with Case A.a are not displayed here but they are lower than those in (12) too.
} 
$I C_{H L v s L L}$, together with $P C_{L L}$. Solving for the wage rates, substituting them into the principal's objective function and maximizing with respect to effort levels (omitting $e_{L L}$ which is already determined) yields

$$
e_{L H}=1+\gamma \quad e_{H H}=\frac{1+\gamma}{2 \theta-1} \quad e_{L L}=1 \quad e_{H L}=\frac{1}{\theta} .
$$

This candidate solution exists for $\theta<\frac{3}{2}$ and $\gamma<\gamma_{M}=2(\theta-1)$, where inequality $\gamma<\gamma_{M}$ is equivalent to the monotonicity condition $e_{H H}>e_{L L}$ and where $\gamma_{M}<1$ whenever $\theta<\frac{3}{2}$. The outside option of type $H L$ is $U_{H L}^{V}=w_{H L}-\frac{1}{2} \theta e_{H L}^{2}$. Substituting for $w_{H L}$ from the binding constraint $I C_{H L v s L L}$ one gets $U_{H L}^{V}=\frac{1}{2} \theta e_{H L}^{2}+\frac{(2-\theta)}{2}-\frac{1}{2} \theta e_{H L}^{2}=\frac{(2-\theta)}{2}$. Hence the difference in reservation utilities for non-motivated types is equal to $U_{L L}^{T S, V}-U_{H L}^{V}=\frac{1}{2}-\frac{(2-\theta)}{2}=\frac{(\theta-1)}{2}$ and this solution is compatible with $P N V$ being in Case 5 for $U_{L L}^{T S, V}-U_{H L}^{V}=\frac{(\theta-1)}{2}>\frac{k^{2}(\theta-1)}{2(2-\theta)^{2}}$ or else for

$$
k<(2-\theta)=k_{4}
$$

where $k_{4}<1$ always holds. So this solution can be discarded.

Pooling between non-motivated types Suppose that optimal effort levels are such that $e_{L H}>$ $e_{H H}>e_{L L}=1=e_{H L}$. The binding constraints are the downward incentive compatibility ones $I C_{L H v s H H}, I C_{H H v s L L}$, together with $P C_{L L}$. Optimal effort levels are given by

$$
e_{L H}=1+\gamma \quad e_{H H}=\frac{1+\gamma}{2 \theta-1} \quad e_{L L}=e_{H L}=1
$$

The outside option for type $H L$ is equal to $U_{H L}^{V}=w_{H L}-\frac{1}{2} \theta e_{H L}^{2}=\frac{(2-\theta)}{2}$ and is the same as in the previous case. Hence, as before, the difference in reservation utilities $U_{L L}^{V}-U_{H L}^{V}=\frac{(\theta-1)}{2}$ is not compatible with the bounds that define Case 5 .

\section{C.3.2 Pooling between intermediate types}

Suppose that optimal effort levels are ordered as $e_{L H}>e_{H H}=e_{L L}=1>e_{H L}$. Now the constraints that one assumes to be binding are $I C_{L H v s H H}, P C_{L L}$ and $I C_{H L v s L L}$ yielding optimal effort levels

$$
e_{L H}=1+\gamma \quad e_{H H}=e_{L L}=1 \quad e_{H L}=\frac{1}{\theta} .
$$

But the difference in reservation utilities $U_{L L}^{V}-U_{H L}^{V}$ is still the same as in the preceding regimes and thus this solution can be discarded because it is not compatible with the bounds delimiting Case 5 for $P N V$.

\section{C.3.3 Ability prevails}

Suppose that, in the mission-oriented sector, ability prevails and that the ordering of effort levels is such that $e_{L H}>e_{L L}=1>e_{H H} \geq e_{H L}$. 
Full separation of types Now the only possible set of binding constraints is $I C_{L H v s L L}, P C_{L L}$, $I C_{H H v s L L}$ and finally $I C_{H L v s H H}$. Optimal effort levels are given by

$$
e_{L H}=1+\gamma \quad e_{L L}=1 \quad e_{H H}=\frac{(2 \gamma+1)}{2 \theta} \quad e_{H L}=\frac{1}{\theta}
$$

where $e_{H H}$ is upward distorted. This solution exists when the monotonicity condition $e_{L L}>e_{H H}$ is satisfied, namely when $\gamma<\frac{2 \theta-1}{2}$. The reservation utility of type $H L$ is equal to $U_{H L}^{V}=\frac{(1-2 \gamma)(1+2 \gamma)+4 \theta(2 \gamma-\theta+2)}{8 \theta}$ and thus the difference in reservation utilities becomes $U_{L L}^{V}-U_{H L}^{V}=\frac{1}{2}-\frac{(1-2 \gamma)(1+2 \gamma)+4 \theta(2 \gamma-\theta+2)}{8 \theta}=$ $\frac{4 \theta(\theta-1-2 \gamma)-(1-2 \gamma)(1+2 \gamma)}{8 \theta}$ which is lower than in the preceding cases and thus not compatible with the bounds delimiting Case 5 for $P N V$.

Finally note that, when $P N V$ is in Case 5, it is never optimal for the vocational principal to offer the null contract to type $H L$. Indeed, this type would always have an incentive to take the contract offered by $P V$ to type $L L$ and then $I C_{H L v s L L}^{V}$ would always be violated.

Therefore, Case 5 for $P N V$ can never be attained in equilibrium when principals compete and $1 \leq$ $k \leq 1+\gamma$.

\section{Wage differentials and the power of incentives}

Depending on the different combinations of states of the world for the two principals, different wages characterize the optimal contracts. Let us consider each possible combination in turn.

Let us start with Situation (i) of Section 5.3. The non-vocational principal is in Case 1 and offers wages

$$
w_{L L}^{N V}=w_{L H}^{N V}=\frac{(2 \theta-1)^{2}+\theta^{2} k^{2}(4 \theta-3)}{2 \theta(2 \theta-1)^{2}} \quad w_{H L}^{N V}=w_{H H}^{N V}=\frac{(2 \theta-1)^{2}+k^{2} \theta^{2}}{2 \theta(2 \theta-1)^{2}}
$$

while the vocational principal offers pooling contracts to non-motivated types and optimal wages offered to motivated types are

$$
w_{L H}^{V}=\frac{\theta(2 \theta-1)^{2}(1-\gamma)(1+\gamma)+\theta(\theta-1)(1+\gamma)^{2}+(1+2 \gamma)(2 \theta-1)^{2}}{2 \theta(2 \theta-1)^{2}} \quad w_{H H}^{V}=\frac{\theta^{2}(1+\gamma)^{2}-2 \gamma \theta(1+\gamma)(2 \theta-1)+(1+2 \gamma)(2 \theta-1)^{2}}{2 \theta(2 \theta-1)^{2}} .
$$

Then type $H H$ gets a lower wage in the mission-oriented sector if and only if $w_{H H}^{V}<w_{H H}^{N V}$ that is if and only if

$$
k>\frac{\sqrt{\left(2 \gamma-6 \theta \gamma+\theta^{2}+2 \theta \gamma^{2}+6 \theta^{2} \gamma-3 \theta^{2} \gamma^{2}\right)}}{\theta}=k_{5},
$$

where $\bar{k}<k_{5}<1+\gamma$. As for type $L H$ we have $w_{L H}^{V}<w_{L H}^{N V}$ if and only if

$$
k>\quad \sqrt{\frac{\left(2 \gamma-10 \theta \gamma-3 \theta^{2}+4 \theta^{3}-2 \theta \gamma^{2}+10 \theta^{2} \gamma+5 \theta^{2} \gamma^{2}-4 \theta^{3} \gamma^{2}\right)}{\theta^{2}(4 \theta-3)}}=k_{6}
$$

with $k_{6}<k_{5}$. Hence the wage gap is easier to observe for motivated workers with high-ability rather than with low-ability. Moreover, $k_{6}<\bar{k}$ for $\gamma<\frac{\left(5 \theta^{2}-5 \theta+1\right)-(2 \theta-1) \sqrt{28 \theta^{3}-16 \theta^{2}-12 \theta^{4}+1}}{\theta\left(4 \theta^{2}-5 \theta+2\right)}=\gamma_{6}$ where $\gamma_{6}>\bar{\gamma}$. Then, 
for sufficiently low motivation, that is for $\bar{\gamma} \leq \gamma<\gamma_{6}$, high-ability motivated workers always experience an earnings penalty, independent of $k$. As for the power of incentives, we have $w_{L H}^{V}-w_{H H}^{V}<w_{L L}^{N V}-w_{H L}^{N V}$ iff $k>\sqrt{\frac{(1+\gamma)(\theta-\gamma(\theta-1))}{\theta}}=k_{7}$, where $k_{7}<\bar{k}$ always holds. Hence we always observe low-powered incentives in the vocational sector in Situation $(i)$.

Suppose now that we are in Situation (ii) of of Section 5.3.

When ability prevails for $P V$ and Case A.a holds, while $P N V$ is in Case 3, then wages in the nonvocational sector are such that

$$
w_{L L}^{N V}=w_{L H}^{N V}=\frac{k^{2}+1}{2} \quad w_{H L}^{N V}=w_{H H}^{N V}=\frac{k^{2}+1}{2 \theta}
$$

while wages in the mission-oriented sector are such that

$$
w_{L H}^{V}=\frac{2 \gamma+2-\gamma^{2}}{2} \quad w_{H H}^{V}=\frac{2 \gamma+2-\gamma^{2}}{2 \theta} .
$$

Then, motivated types earn less in the mission oriented sector where they choose to work (irrespective of their ability) if and only if

$$
k>\sqrt{1+\gamma(2-\gamma)}=k_{8},
$$

where $k_{8}<\underline{k}$ for $\gamma<\gamma_{8}=1-\sqrt{(2-\theta)}$, with $\gamma_{A}>\gamma_{8}>\gamma_{P}$. Hence, when $P V$ is in Case A.a, one observes the wage differential for $k_{8}<k<\underline{k}$. As for the power of incentives, one has $w_{L H}^{V}-w_{H H}^{V}<w_{L L}^{N V}-w_{H L}^{N V}$ iff $k_{8}<k<\underline{k}$, namely low-powered incentives are in place in the vocational sector precisely under the same conditions under which an earnings penalty emerges.

When $P N V$ is in Case 2 and $\underline{k} \leq k<\bar{k}$ while $P V$ is still in Case A.a, the only wage that changes with respect to expressions (18) and (19) is $w_{H H}^{N V}$ which becomes lower and equal to $w_{H H}^{N V}=\frac{\theta+1}{2 \theta}$. Now, motivated types always earn less in the mission-oriented sector and the wage differential is always in place.

Low-powered incentives are also offered in the mission-oriented sector, because $w_{L H}^{V}-w_{H H}^{V}<w_{L L}^{N V}-$ $w_{H L}^{N V}$ holds iff $k>\sqrt{\frac{(\theta-1)\left(2 \gamma+2-\gamma^{2}\right)+1}{\theta}}=k_{9}$ but $k_{9}<\underline{k}$, so inequality $k>k_{9}$ is always satisfied in this case.

Suppose now that ability prevails for $P V$ and Case $A . b$ holds while $P N V$ is in Case 3 , then wages are the same as in expressions (18) and (19) except for $w_{H H}^{V}$ which increases to $w_{H H}^{V}=\frac{2 \theta+1-(\theta-\gamma)^{2}}{2 \theta}$. We observe a wage gap for type $L H$ only when $\gamma_{P}<\gamma<\gamma_{8}$ and $k_{8}<k<\underline{k}$ but the wage gap never exists for type $H H$. Low-powered incentives are offered by the vocational principal iff $\sqrt{\left(\theta-\gamma^{2}\right)}=k_{10}<k<\underline{k}$. If instead $P N V$ is in Case 2 then the pay penalty is in place for type $L H$ when $\gamma_{P}<\gamma<\gamma_{8}$, or when $\gamma_{8} \leq \gamma<\gamma_{A}$ and $k_{8}<k<\bar{k}$ occur whereas the pay gap exists for type $H H$ when $\gamma_{P}<\gamma<\theta-\sqrt{\theta}=$ $\gamma_{9}<\gamma_{8}$. And low-powered incentives are offered by the vocational principal iff $k>\sqrt{\frac{\left(\theta^{2}-(\theta-1) \gamma^{2}\right)}{\theta}}=k_{11}$; but $k_{11}<\underline{k}$ therefore low-powered incentives are always offered when $P N V$ is in Case 2 and $P V$ in Case A.b. 
When $P V$ offers pooling contracts to types $L L$ and $H H$, wages in the vocational sector are

$$
w_{L H}^{V}=\frac{2 \gamma+2-\gamma^{2}}{2} \quad w_{H H}^{V}=1
$$

Then, irrespective of whether $P N V$ is in Case 2 or 3, type $H H$ is always paid more in the vocational sector, while the wage differential still exists for type $L H$ provided that $k_{8}<k<\bar{k}$. As for the power of incentives, low-powered incentives are always in place when $P N V$ is in Case 2 because the necessary and sufficient condition is $k>\sqrt{\frac{1+(2-\gamma) \theta \gamma}{\theta}}=k_{12}$ and $k_{12}<\underline{k}$. Finally, low-powered incentives are in place when $P N V$ is in Case 3 iff $\sqrt{\frac{(2-\gamma) \gamma \theta-(\theta-1)}{(\theta-1)}}=k_{13}<k<\underline{k}$.

To conclude, suppose that motivation prevails for $P V$ so that wages in the vocational sector are

$$
w_{L H}^{V}=\frac{(1-\gamma)(1+\gamma)(2 \theta-1)^{2}+(\theta-1)(1+\gamma)^{2}+(2 \gamma+2-\theta)(2 \theta-1)^{2}}{2(2 \theta-1)^{2}} \quad w_{H H}^{V}=\frac{(1+\gamma)(\theta+2 \gamma-3 \theta \gamma)+(2 \gamma+2-\theta)(2 \theta-1)^{2}}{2(2 \theta-1)^{2}}
$$

Again, irrespective of whether $P N V$ is in Case 2 or 3, both types $H H$ and $L H$ are always paid more in the vocational sector, and the wage differential does not exist. Now, low-powered incentives are never offered in the mission-oriented sector when $P N V$ is in Case 2, while they do arise for $k_{14}=$ $\frac{\sqrt{4 \theta(\gamma+\theta-\theta \gamma)(\gamma+1)-(2 \theta-1)^{2}}}{(2 \theta-1)}<k<\underline{k}$ when $P N V$ is in Case 3 .

\section{References}

[1] Armstrong M. (1996), "Multiproduct nonlinear pricing", Econometrica, 64, 51-75.

[2] Armstrong M. and J-J. Rochet (1999), "Multi-dimensional Screening: A User's Guide”, European Economic Review, 43, 959-979.

[3] Armstrong M. (1999), "Optimal Regulation with Unknown Demand and Cost Functions", Journal of Economic Theory 84, 196-215.

[4] Bargain, O. and B. Melly (2008): "Public Sector Pay Gap in France: New Evidence Using Panel Data", IZA Discussion Paper n. 3427.

[5] Barigozzi F. and G. Turati (2012), "Human Health Care and Selection Effects. Understanding Labour Supply in the Market for Nursing", Health Economics 21(4), 477-483.

[6] Barigozzi F., N. Burani and D. Raggi (2013), "The Lemons Problem in a Labor Market with Intrinsic Motivation. When Higher Salaries Pay Worse Workers", working paper n. 883, Department of Economics, University of Bologna.

[7] Barigozzi F. and N. Burani (2013), "Bidimensional Screening with Intrinsically Motivated Workers", working paper n. 866, Department of Economics, University of Bologna.

[8] Basov S. (2005), Multidimensional Screening, Springer-Verlag. 
[9] Basov S. (2001), "Hamiltonian Approach to Multidimensional Screening" Journal of Mathematical Economics, 36, 77-94.

[10] Bénabou R. and J. Tirole (2013), "Bonus Culture: Competitive Pay, Screening, and Multitasking", NBER Working Paper No. 18936.

[11] Besley T. and M. Ghatak (2005), "Competition and Incentives with Motivated Agents", American Economic Review 95(3), 616-636.

[12] Biglaiser G. and C. Mezzetti (1993), "Principals Competing for an Agent in the Presence of Adverse Selection and Moral Hazard", Journal of Economic Theory 61, 309-330.

[13] Biglaiser G. and C. Mezzetti (2000), "Incentive Auctions and Information Revelation", Rand Journal of Economics 31(1), 145-164.

[14] Delfgaauw J. and R. Dur (2007), "Signaling and screening of workers' motivation", Journal of Economic Behavior and Organization 62, 605-624.

[15] Delfgaauw J. and R. Dur (2008), "Incentives and workers' motivation in the public sector", The Economic Journal 118, 171-191.

[16] Delfgaauw J. and R. Dur (2010), "Managerial talent, motivation, and self-selection into public management", Journal of Public Economics 94(9-10), 654-660.

[17] Deneckere R. and S. Severinov (2011), "Multi-Dimensional Screening: A Solution to a Class of Problems", mimeo, econ.ucsb.edu.

[18] Disney, R. and A. Gosling (1998), "Does it Pay to Work in the Public Sector?" Fiscal Studies 19, $347-374$.

[19] Gibbons R. and L. Katz (1992), "Does Unmeasured Ability Explain Inter-Industry Wage Differentials?", Review of Economic Studies 59, 515-535.

[20] Goddeeris J.H. (1988), "Compensating Differentials and Self-Selection: An Application to Lawyers", Journal of Political Economy 96(2), 411-428.

[21] Goux D. and E. Maurin (1999), "Persistence of Interindustry Wage Differentials: A Reexamination Using Matched Worker-Firm Panel Data", Journal of Labor Economics 17(3), 492-533.

[22] Gregg, P., P. Grout, A. Ratcliffe, S. Smith, and F. Windmeijer, (2011), "How important is pro-social behaviour in the delivery of public services?", Journal of Public Economics 95(7-8), 758-766.

[23] Handy F. and E. Katz (1998), "The wage differential between nonprofit institutions and corporations: getting more by paying less?", Journal of Comparative Economics 26, 246-261.

[24] Heyes A. (2005) "The economics of vocation or -why is a badly paid nurse a good nurse-?", Journal of Health Economics 24, 561-569. 
[25] Hwang H., W.R. Reed and C. Hubbard (1992), "Compensating Wage Differentials and Unobserved Productivity", Journal of Political Economy 100(4), 835-858.

[26] Laffont J-J. and D. Martimort (2002), The theory of Incentives: The Principal-Agent Model, Princeton University Press.

[27] Melly, B. (2005), "Public-private wage differentials in Germany: evidence from quantile regression", Empirical Economics 30(2), 505-520.

[28] Mocan, N., E. Tekin (2003), "Nonprofit sector and part-time work: an analysis of employer-employee matched data of child care workers", Review of Economics and Statistics 85(1), 38-50.

[29] Murdock K. (2002), "Intrinsic Motivation and Optimal Incentive Contracts", RAND Journal of Economics, 33(1), 650-671.

[30] Prendergast C. (2007), "The Motivation and Bias of Bureaucrats", American Economic Review 97(1), 180-196.

[31] Prendergast C. (2008), "Intrinsic Motivation and Incentives", American Economic Review: Papers E Proceedings 98(2), 201-205.

[32] Preston, A. (1989), "The nonprofit worker in a for-profit world", Journal of Labor Economics 7, 438-463.

[33] Rochet J.-C. and P. Chonè (1998), "Ironing, Sweeping, and Multidimensional Screening" Econometrica, 66, 783-826.

[34] Roomking M. and B. Weisbrod (1999): "Managerial Compensation and Incentives in For-Profit and Nonprofit Hospitals", Journal of Law, Economics and Organization, 15(3), 750-781.

[35] Rosen, S. (1986), "The Theory of Equalizing Differences", in: Ashenfelter, O., Layard, R. (Eds.), Handbook of Labor Economics, vol. 1, North Holland, pp. 641-692.

[36] Stole L.A. (1995), "Nonlinear pricing and oligopoly", Journal of Economics $\& 3$ Management Strategy 4, 529-562. 


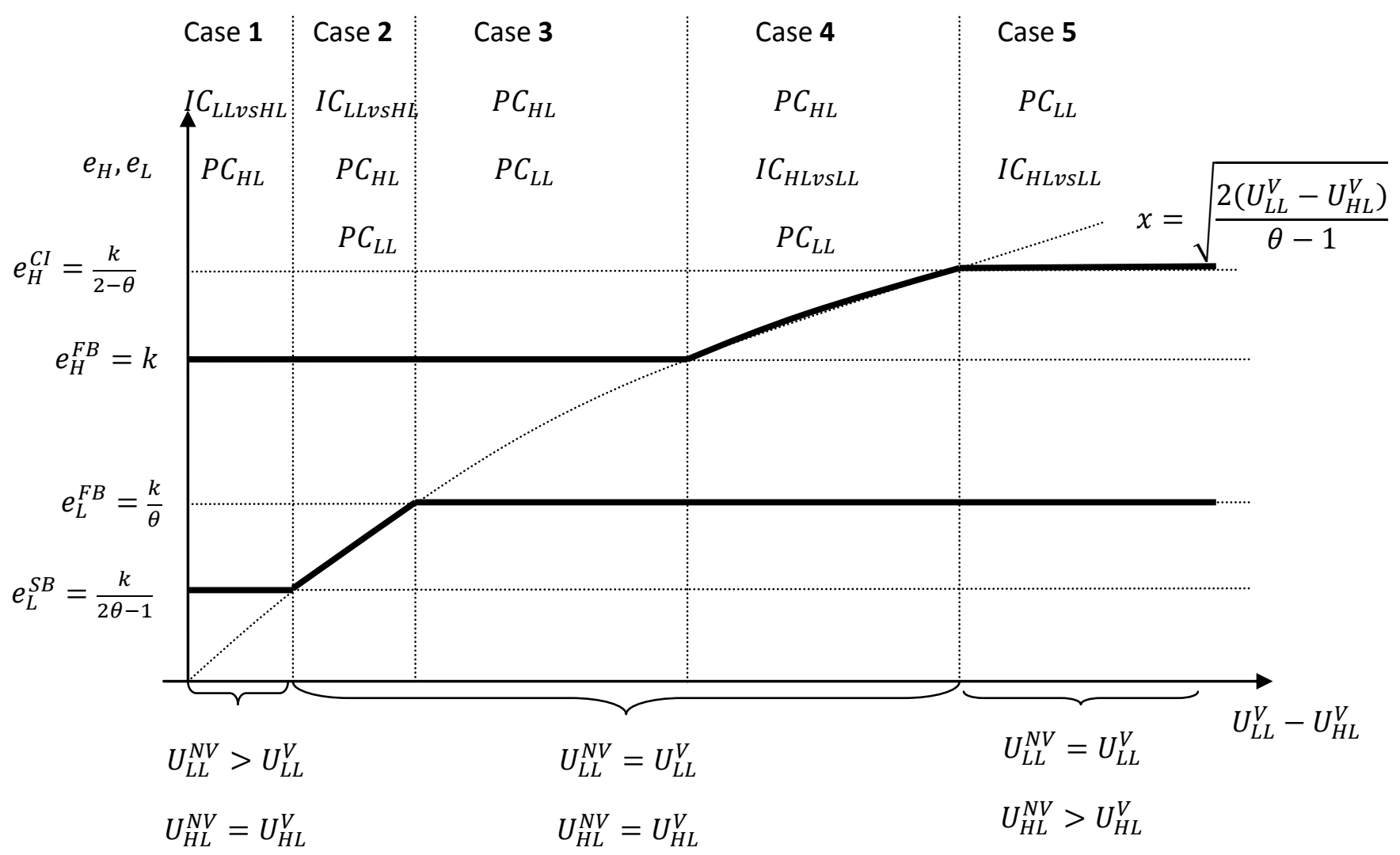

Figure 1: Reaction function of principal NV when $1 \leq \mathrm{k} \leq 1+\gamma$. 


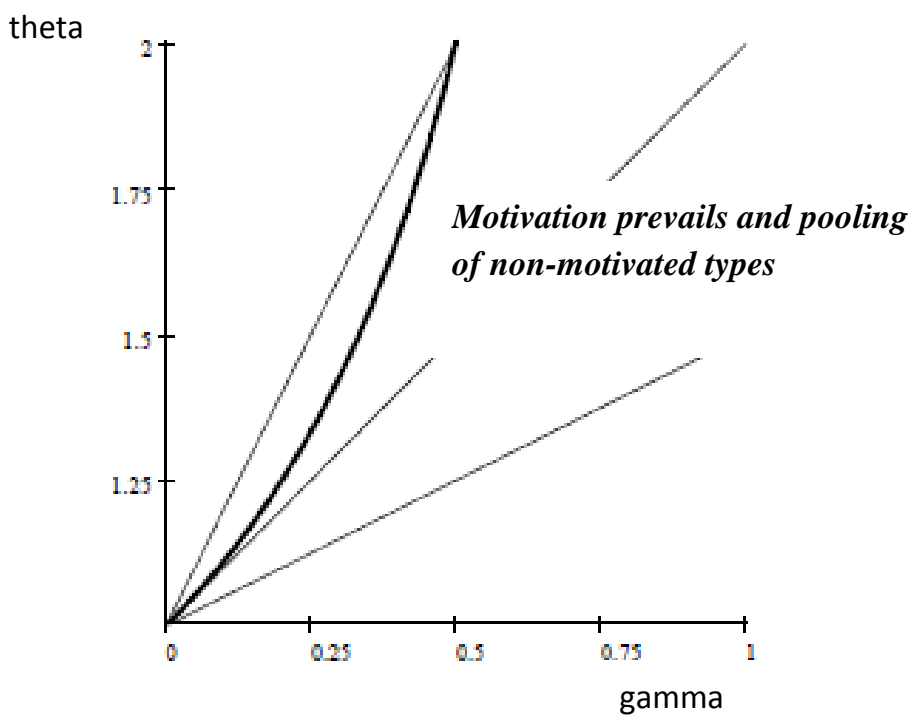

Figure 2a: Case (i) for principal V. 


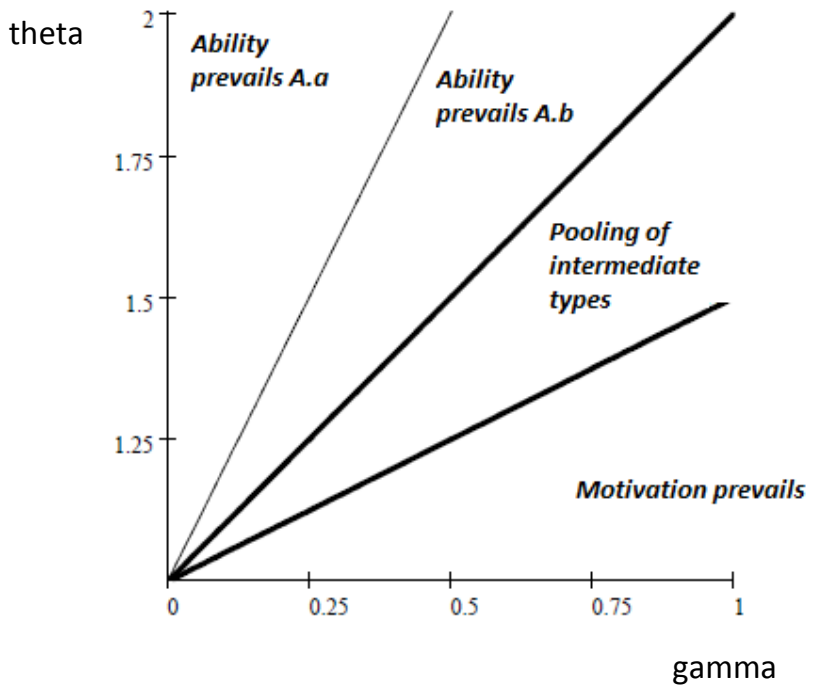

Figure 2b: Case (ii) for principal V. 


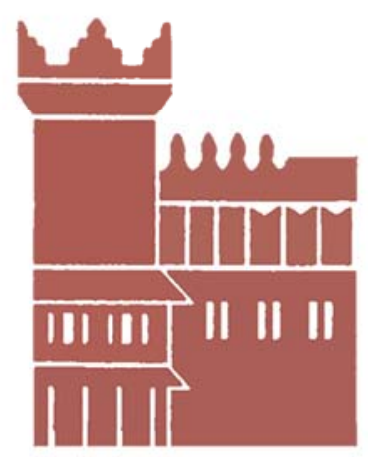

Alma Mater Studiorum - Università di Bologna DEPARTMENT OF ECONOMICS

Strada Maggiore 45

40125 Bologna - Italy

Tel. +39051 2092604

Fax +390512092664

http://www.dse.unibo.it 